\title{
Platonic Triangles of Groups
}

\author{
Roger C. Alperin
}

\section{CONTENTS}

1. Introduction to Triangles of Groups

2. Homological Restrictions

3. Platonic Restrictions

4. Vertex Data

5. Negatively Curved Geometric Platonic Groups

6. Flat Platonic Groups

References

Research supported by NSA and NSF.

1980 Mathematics Subject Classification (1985 Revision): $20 \mathrm{E} 99$.

Keywords and Phrases: triangle of groups, Coxeter group, FA, cohomological dimension.
Non-positively curved triangles of finite groups are of cohomological dimension 2 over the rationals and have Property FA. We classify triangles of finite groups which satisfy certain geometric conditions including the Gauss-Bonnet theorem. We investigate whether or not these groups are virtually torsionfree, contain a free abelian subgroup of rank 2, are residually finite or are linear.

In this article we classify a collection of groups that have characteristics in common with certain hyperbolic Coxeter groups. The classification of hyperbolic reflection groups in dimension three has been known since Lannér's thesis in 1950 [Coxeter and Moser 1980]. There are 9 co-compact and 28 noncompact finite volume discrete subgroups of hyperbolic 3-space [Humphreys 1990], all with quotient a single simplex. The noncompact groups have Euler characteristic zero by duality; see [Serre 1970]. Any hyperbolic reflection group is a finitely generated complex linear group, and so is residually finite and virtually torsion-free [Alperin 1987]. Furthermore, in the noncompact case, the link of an ideal vertex, of the tesselation by tetrahedra, is a (flat horospherical) plane with a free abelian rank 2 group of symmetries. In fact, there is always a subdiagram of the Coxeter diagram having the shape $\bullet^{4} \bullet{ }^{4} \bullet, \bullet \bullet \bullet$, , or $\boldsymbol{\bullet}^{6}$ and giving rise to the virtually free abelian subgroup. Other interesting information on certain hyperbolic Coxeter groups can be found in [Milnor 1994], which considers the volumes of those 10 groups with a "straight line" Coxeter diagram corresponding to the orthosimplex condition.

When the Coxeter group has an ideal vertex, we can retract the complex onto a contractible 2 complex, which is the barycentric subdivision of 
the Coxeter complex retracted away from the vertices at infinity of the tetrahedral packing [Alperin 1980]. Now, the rotation subgroup of index 2 in the Coxeter group has a particularly nice presentation [Brunner et al. 1985] as a triangle of groups in terms of its triangle fundamental domain. Moreover, these groups satisfy a form of the GaussBonnet Theorem based on the angular defect of this triangle. There are 9 of the 28 noncompact finite volume hyperbolic manifolds for which all the vertex stabilizers are finite. The remainder have some of their vertex stabilizers of Euler characteristic zero, that is virtually free abelian of rank 2 . Upon retraction of the Coxeter complex onto its associated 2-complex, the flat horospherical plane survives.

Here we shall classify the (minimal) groups that have a triangle of groups decomposition satisfying a Gauss-Bonnet condition, for which the Euler characteristic is zero and the vertex stabilizers are finite. Our less restrictive conditions allow more families of our so called Platonic groups. Imposing certain "geometric" conditions, we obtain a classification very nearly the same as the rotation subgroups of hyperbolic reflection groups described above. These are the spherical or toroidal flat Platonic groups. We also determine here the geometric Platonic groups that are negatively curved. Many of these are also Coxeter groups; they are not hyperbolic in the Coxeter group sense but, in fact, do act on hyperbolic space yielding an infinite volume hyperbolic orbifold.

Our main results, stated roughly, are that there are finitely many flat Platonic triangles of finite groups, and that there are finitely many families of nonpositively curved geometric Platonic triangles of finite groups.

I thank Paul Brown for allowing access to his programs and other results of his thesis, and also P. Huneke and H. Glover for discussions on graph embeddings. The coset enumerations and other group-theoretic calculations have been programed using GAP and MAGMA. Using GAP, Brown's program computes the diameter and adjacency relations of the vertex link graphs. These can then be displayed and simplified using the program called Groups\&Graphs2.4.

These results would not have been obtained without a significant degree of experimentation using computer calculations. The organizational features of this investigation only became clear after many computations, and it became possible to formulate the definition of a Platonic triangle of groups. Certainly, the proof that a nonpositively curved triangle of finite groups is virtually torsion-free is still an outstanding problem.

Extending these ideas to classify "nongeometric" Platonic triangles of groups seems daunting, if not impossible, without further theoretical results. The flat Platonic triangles, however, seem accessible. Also, it would be very interesting to determine the graph-theoretic restrictions necessary for a finite graph to have a finite cover that is planar. Moreover, making the connection of the geometric Platonic groups with the (orbifold) fundamental group of special 3-dimensional manifolds is a fascinating open problem.

\section{INTRODUCTION TO TRIANGLES OF GROUPS}

We first review the basic ideas and theorems about triangles of groups proved by Gersten and Stallings [Stallings 1991]. Fundamental to the investigation of triangles of groups is the angle at a vertex of the triangle. If we have groups $E$ and $F$ with a "common" subgroup $D$ and injective homomorphisms $E \rightarrow A, F \rightarrow A$ agreeing on $D$, the angle at group $A$ between $E$ and $F$ (along $D$ ) is defined as $\frac{\pi}{r}$, where $2 r$ is the length of the shortest alternating word, $e_{1} f_{1} e_{2} f_{2} \cdots e_{r} f_{r}$ with $e_{i} \in E-D$ and $f_{i} \in F-D$, that lies in the kernel of the induced homomorphism $E_{D}^{*} F \rightarrow A$. A triangle of groups is the universal group given by vertex, edge and face data as follows: vertex groups $A, B, C$ and edge groups $E, F, G$ with a common face group $D$, together with homomorphisms to $A, B, C$ such that the angle at $A$ between $E$ and $F$ (along $D$ ) is $\frac{\pi}{r}$, 
the angle at $B$ between $F$ and $G$ (along $D$ ) is $\frac{\pi}{s}$, the angle at $C$ between $E$ and $G$ (along $D$ ) is $\frac{\pi}{t}$. The triangle group

$$
\Gamma(\mathcal{T})=\Gamma(A, B, C ; E, F, G ; D ; r, s, t)
$$

is the universal group extending the given homomorphisms with associated triangle $\mathcal{T}$ having angles $\left[\frac{\pi}{r}, \frac{\pi}{s}, \frac{\pi}{t}\right]$. The curvature characteristic of the triangle, $\kappa_{\mathcal{T}}$, is defined as $\frac{1}{r}+\frac{1}{s}+\frac{1}{t}-1$. A triangle is nonpositively curved if $\kappa_{\mathcal{T}} \leq 0$. Gersten and Stallings showed that if the triangle group is associated to a nonpositively curved triangle then there is a contractible two-complex on which $\Gamma(\mathcal{T})$ acts with a single triangle as fundamental domain having vertex, edge and face stabilizers as given by the data; in particular, vertex, edge and face groups inject in the triangle group. Moreover, any bounded subgroup (and in particular any finite subgroup) of $\Gamma(\mathcal{T})$ is conjugate to a subgroup of one of the vertex stabilizers.

If the triangle is negatively curved and all the vertex groups are finite, the Corollary to Theorem A of [Bridson 1995] implies that either the group $\Gamma$ is word hyperbolic and hence any abelian subgroup is virtually cyclic or the 2-complex of GerstenStallings contains a flat plane; furthermore, these conditions are mutually exclusive. Moreover, if the group is word hyperbolic, Sela [Sela 1993] has shown that it is Hopfian. For general finitely generated groups, residually finite implies Hopfian. By the remarks above, there are only finitely many conjugacy classes of elements of finite order in a triangle of finite groups; in such situations, residually finite groups are virtually torsion-free. We might expect that nonpositively curved triangles of finite groups are residually finite; however, this is not generally true as has been recently shown by Hsu and Wise [1998].

The automatic nature and bicombings of triangles of finite groups have been studied in [Floyd and Parry 1997; Noskov 1995]. Also, Wise (unpublished) has exhibited $F_{2} \times F_{2}$ as a subgroup in a certain triangle of finite groups, consequently showing that triangles of finite groups are not necessarily coherent.

For our flat geometric Platonic groups, we show that the groups are virtually torsion-free. In the case of hyperbolic Coxeter groups of Euler characteristic zero, there is a free abelian subgroup of rank 2 as discussed above. Our classification indicates that the associated 2-complex to a flat Platonic group is almost always the same as the 2complex for one of the hyperbolic Coxeter group. Thus, there is an isometric flat plane and consequently, by results of P. Brown [1997], there is a rank-2 free abelian subgroup. Thus, none of these cases yield word hyperbolic groups. It would appear then that the distinct Platonic groups that act on the same 2-complex may just be different lattices in the automorphism group of the complex. We hope to pursue these issues in a subsequent paper.

\section{HOMOLOGICAL RESTRICTIONS}

A group $G$ is said to have Property FA if whenever it acts (without inversions) on a tree then there is a point fixed by all of $G$. In [Alperin 1996] we showed that a triangle of finite groups has Property FA. This can be generalized as follows. We shall call a triangle of groups minimal if each of the vertex groups is generated by its associated edge groups.

Theorem 2.1. Suppose that $\Gamma=\Gamma(\mathcal{T})$ is a minimal nonpositively curved triangle of groups so that all edge groups properly contain the face group and such that all orders of elements of finite order in $\Gamma$ are invertible in the ring $R$. If each of the vertex stabilizers has Property $F A$ and $\operatorname{cd}_{R} \leq 2$ and each of the edge stabilizers has $\mathrm{cd}_{R} \leq 1$, then $\Gamma$ has Property FA and $\operatorname{cd}_{R}(\Gamma)=2$.

Proof. It follows immediately from the spectral sequence (Quillen's Lemma [Serre 1971]) for the action of $\Gamma$ on the contractible 2-complex $X$ constructed by Gersten and Stallings [Stallings 1991] that $\operatorname{cd}_{R}(\Gamma) \leq 2$. Now if $\operatorname{cd}_{R}(\Gamma) \leq 1$, then equivalently by [Dicks and Dunwoody 1989, Theorem 
3.13] there is a nontrivial action on a tree $\mathcal{W}$ (without inversions), having finite vertex stabilizers of order invertible in $R$. Since the vertex groups, $A$, $B$ and $C$, of $\Gamma$ have Property FA, they fix the vertices $x, y$ and $z$ of $\mathcal{W}$. If these vertices are distinct, then consider a common point $P$ in $y z \cap x z \cap x y$. The segment $y z$ is fixed by $F, x z$ is fixed by $G$ and $x y$ is fixed by $E$. Thus, since $\Gamma$ is generated by its edge groups, the common point $P$ is fixed by $\Gamma$ and hence $\Gamma$ is finite since the action on the tree has finite stabilizers. Also, if there is a single vertex $x=y=z$, or if these vertices are reduced to two, say $x \neq y=z$, then since $B, C$ stabilize $y$, the group $\Gamma$ stabilizes $y$ since it is generated by the edge groups. Thus $\Gamma$, being finite, fixes a vertex for its action on the 2 complex $\mathcal{X}$ by the theorem of Gersten andStallings. By a conjugation then say $\Gamma \subset A$ and also the opposite edge group $G \subset \Gamma$ so that $\Gamma$ stabilizes the entire triangle fundamental domain, and hence is contained in $D$. This is a contradiction, so the cohomological dimension is 2 .

Corollary 2.2. Suppose that $\Gamma=\Gamma(\mathcal{T})$ is a nonpositively curved triangle of finite groups so that all edge groups properly contain the face group and such that all orders of elements of finite order in $\Gamma$ are invertible in the ring $R$, then $\operatorname{cd}_{R}(\Gamma)=2$.

Proof. Consider the group $\Gamma_{1}$ generated by the edge groups of $\Gamma$; it is a minimal triangle of groups with the same angles as $\Gamma$. By Quillen's Lemma [Serre 1971], $\operatorname{cd}_{R}(\Gamma) \leq 2$. From the theorem, $\Gamma_{1}$ has cohomological dimension 2 , and thus $\operatorname{cd}_{R}(\Gamma) \geq$ $\operatorname{cd}_{R}\left(\Gamma_{1}\right)=2$.

Thus, it follows that a nonpositively curved triangle of finite groups has no free abelian subgroups of rank greater than 2. Note that triangle groups are examples of groups with FA and $v c d_{R}=2$. An infinite group of $v c d_{R}=1$ does not have Property FA.

Observe also that if one of the edge groups is trivial, then $\Gamma$ is a free product with amalgamation. We can realize the group as the graph of groups $B \underset{E}{\underset{F}{*}} A \underset{F}{*} C$, if $G=\{1\}$. If, moreover, the vertex groups are finite, then the group is virtually free and hence of virtual cohomological dimension $\leq 1$.

Question 2.3. A nonpositively curved triangle of free groups is of cohomological dimension at most 2 , if the face group is trivial. Is it of cohomological dimension 2 if the angles are all nonzero $\Gamma$

Question 2.4. Can one describe in group theoretic terms a nonpositively curved triangle of groups that has Property FA and is of cohomological dimension 2 over a ring $R \Gamma$ Theorem 2.1 describes some of these groups, but one can now iterate this procedure to get more complicated groups.

It follows from [Serre 1971] that a triangle of finite groups is of type VFL if it is virtually torsionfree; also, if it is virtually torsion-free, then it has an Euler-Wall characteristic. If the Euler-Wall characteristics exist for the vertex, edge and face groups, we define the orbifold characteristic as

$$
\begin{aligned}
\chi(\Gamma(\mathcal{T}))=\chi(A)+ & \chi(B)+\chi(C) \\
& -\chi(E)-\chi(F)-\chi(G)+\chi(D) .
\end{aligned}
$$

This agrees with the Euler-Wall characteristic of $\Gamma$, if it exists.

\section{PLATONIC RESTRICTIONS}

Our interest is in the situation where the triangle group data satisfies the following conditions:

(i) The triangle is nonpositively curved, with all nonzero angles.

(ii) All vertex groups have nonnegative Euler characteristic.

(iii) All edge groups are nontrivial and $D=\{1\}$.

(iv) The triangle group data is minimal.

(v) $2 \chi(\Gamma(\mathcal{T}))=\kappa_{\mathcal{T}}$.

When all these conditions are satisfied we call $\Gamma(\mathcal{T})$ a Platonic group. If, furthermore, the triangle $\mathcal{T}$ is a Euclidean triangle, we call this a flat Platonic group. If all the vertex groups are finite, we call it is a Platonic triangle of finite groups.

We shall, in fact, assume for the rest of this article that all vertex groups are finite. Properties (i), 
(ii) and (iii) guarantee cohomological dimension 2 as we have shown above. Property $(v)$ is a fake Gauss-Bonnet condition. Notice that, if $|A|=a$, $|B|=b$, etc., we have

$$
\chi(\Gamma(\mathcal{T}))=\frac{1}{a}+\frac{1}{b}+\frac{1}{c}-\frac{1}{e}-\frac{1}{f}-\frac{1}{g}+1 .
$$

Thus, $\chi(\Gamma(\mathcal{T}))$ can only be nonpositive if $\frac{1}{e}+\frac{1}{f}+\frac{1}{g}>$ 1 , and this happens for precisely the orders as indicated below in Proposition 3.1. In all other situations, since the curvature characteristic is nonpositive, we will have $2 \chi(\Gamma(\mathcal{T}))>\kappa_{\mathcal{T}}$; so the Platonic situation of condition (v) is in a sense extremal.

Proposition 3.1. If $\chi(\Gamma(\mathcal{T})) \leq 0$, the possibilities for the orders of any edge group (up to a permutation) are

$$
\begin{array}{ll}
{[2,2, n], \text { with } n \geq 2} & (\text { type } D), \\
{[2,3,3]} & (\text { type } T), \\
{[2,3,4]} & (\text { type } O), \\
{[2,3,5]} & (\text { type } I) .
\end{array}
$$

Proof. The condition $\chi(\Gamma(\mathcal{T})) \leq 0$ implies

$$
0<\frac{1}{a}+\frac{1}{b}+\frac{1}{c} \leq \frac{1}{e}+\frac{1}{f}+\frac{1}{g}-1 .
$$

It is well known (and easy to check) that the only solutions for $e, f$ and $g$ to this inequality are those given in the statement of the proposition. The names of the types come from the parallel classification of finite subgroups of $\mathrm{SO}(3)$ : the dihedral, tetrahedral, octahedral, and icosahedral symmetry groups.

Remark. If we don't have just finite vertex groups but still assume nonnegative Euler characteristic of the vertices then the other possible edge configurations are $[2,3,6],[2,4,4]$, and $[3,3,3]$.

Corollary 3.2. For a Platonic group,

$$
\frac{1}{e}+\frac{1}{f}+\frac{1}{g}-1= \begin{cases}\frac{1}{n} & \text { for type } D \\ \frac{1}{6} & \text { for type } T \\ \frac{1}{12} & \text { for type } O \\ \frac{1}{30} & \text { for type } I\end{cases}
$$

and

$$
\frac{1}{a}+\frac{1}{b}+\frac{1}{c}= \begin{cases}\frac{1}{2} \kappa_{\mathcal{T}}+\frac{1}{n} & \text { for type } D \\ \frac{1}{2} \kappa_{\mathcal{T}}+\frac{1}{6} & \text { for type } T \\ \frac{1}{2} \kappa_{\mathcal{T}}+\frac{1}{12} & \text { for type } O \\ \frac{1}{2} \kappa_{\mathcal{T}}+\frac{1}{30} & \text { for type } I\end{cases}
$$

Question 3.3. Which Platonic groups contain a 3manifold group as a subgroup of finite index $\Gamma$

\section{VERTEX DATA}

\section{Numerical Conditions}

We call a group $A$ an amalgam of its subgroups $E$ and $F$ along $D$ if $E, F$ generate $A$ and $E \cap F=$ $D$. Thus for an amalgam the natural surjective homomorphism $\Gamma_{A}=E_{D}^{*} F \rightarrow A$ has a kernel $K_{A}$ that trivially intersects the conjugates of $E$ and $F$. If, moreover, the group $A$ is finite, then we call it a finite amalgam.

For a vertex group $A$ of a negatively curved triangle of groups we assume that $E$ and $F$ properly contain $D, E \cap F=D$, and also that the vertex group is generated by $E$ and $F$. Thus the vertex group is an amalgam of its associated edge groups along the face group. The group $\Gamma_{A}$ has a natural action on a tree constructed as follows. The edges are the right cosets of $D$ in $\Gamma_{A}$ and the edges are of two types, either the cosets of $E$ or $F$ in $\Gamma_{A}$. The incidence relation is $E x$ is joined to $F y$ if $D x=D y$. The group $\Gamma_{A}$ acts on the right on this tree as a group of isometries. Now by the properties of the homomorphism the action of $K_{A}$ on this tree is free and hence is a free group. The quotient of this tree by $K_{A}$ is a bipartite graph $X_{A}$ that can be identified with the graph obtained from the coset construction, as above, applied to $A$, namely its vertices are the cosets of $E$ and $F$ in $A$ and its edges are the cosets of $D$ in $A$. The incidence relation is defined similarly. The angle at vertex $A$ is determined by the length of the shortest cycle in this graph. If $D$ is trivial then the edges of this graph are in one-to-one correspondence with the elements of $A$. 
Now assume that the Platonic conditions (i)-(iv) hold. Consider the vertex at $A$. Suppose that $K_{A}$ has rank $k_{A}$. The graph $X_{A}$, which is bipartite having $|A|=a$ edges; $v_{A}=v_{E}+v_{F}$ vertices, $v_{E}$ of degree $e=|E|, v_{F}$ of degree $f=|F|$ and $e \cdot v_{E}=$ $a=f \cdot v_{F} ; k_{A}$ independent cycles, so that $k_{A}=$ $a-v_{A}+1$. The length of the smallest cycle in this graph is $2 \gamma_{A}$ corresponding to the angle at vertex $A$ of $\frac{\pi}{\gamma_{A}}$. From the Euler characteristic formula we have $\frac{1}{e}+\frac{1}{f}-1=\frac{1-k_{A}}{a}$ or equivalently $\frac{1+k_{A}}{a}=$ $\frac{2}{a}+1-\frac{1}{e}-\frac{1}{f}$. Given a triangle of groups we may add each of these Euler characteristic conditions at the three vertices to obtain the formula

$$
2 \chi(\Gamma(\mathcal{T}))=\frac{1+k_{A}}{a}+\frac{1+k_{B}}{b}+\frac{1+k_{C}}{c}-1 .
$$

We have $\gamma_{A} \geq 2$ since $D=\{1\}$. We assume that $f \leq e$.

Proposition 4.1. We have

$$
\frac{1+k_{A}}{a} \geq \frac{1}{\gamma_{A}}
$$

except in the following cases:

- $\gamma_{A}=2$ and either $2=f<e$ or $3=f \leq e$, or

$-\gamma_{A}=3$ and $2=f<e \leq 5$.

Proof. Since

$$
\frac{1+k_{A}}{a}=\frac{2}{a}+1-\frac{1}{e}-\frac{1}{f}
$$

and $\gamma_{A} \geq 2$, the inequality is easily satisfied for $e, f \geq 4$. Also, if $e=f=2$ then the group is dihedral so $a=2 \gamma_{A}$ and $k_{A}=1$ and we have an equality. Now, if $e, f \geq 3$ and $\gamma_{A} \geq 3$ or if $f=2$, $e \geq 6, \gamma \geq 3$ then the inequality is also obviously satisfied. Furthermore, if $\gamma \geq 2, f=3, e \geq 6$ then the inequality is valid.

Thus, in the generic situation $e, f, g \geq 6$, we have

$$
2 \chi(\Gamma(\mathcal{T})) \geq \kappa_{\mathcal{T}} .
$$

Let $\Gamma(E, F: m)$ denote the set of finite amalgams $A$ of $E$ and $F$ along $\{1\}$ having angle $\frac{\pi}{m}$; let $\Gamma(p, q: m)$ denote the set of all finite amalgams having angle $\frac{\pi}{m}$ where $|E|=p,|F|=q, p \geq q$.
Set $E^{\prime}=E-\{1\}$ and $F^{\prime}=F-\{1\}$.

Proposition 4.2. Suppose $A \in \Gamma(p, q: m)$.

(1) If $m$ is even, the sets $\{1\}, E^{\prime}, F^{\prime}, E^{\prime} F^{\prime}, F^{\prime} E^{\prime}$, $E^{\prime} F^{\prime} E^{\prime}, F^{\prime} E^{\prime} F^{\prime}, \ldots, F^{\prime}\left(E^{\prime} F^{\prime}\right)^{(m / 2)-1},\left(E^{\prime} F^{\prime}\right)^{m / 2}$ are disjoint as subsets of $A$.

(2) If $m$ is odd, the sets $\{1\}, E^{\prime}, F^{\prime}, E^{\prime} F^{\prime}, F^{\prime} E^{\prime}$, $E^{\prime} F^{\prime} E^{\prime}, F^{\prime} E^{\prime} F^{\prime}, \ldots, E^{\prime}\left(F^{\prime} E^{\prime}\right)^{(m-1) / 2}$ are disjoint as subsets of $A$.

Proof. Arguing by contradiction, we may modify a potential overlap of two of these sets, say $w=u$ to $w u^{-1}$ or $u^{-1} w$ and also conjugate if necessary to get an alternating word in $E * F$ that gives a relation in $A$ but of length smaller than $2 m$.

Notice that, if the sets as described in the statement of Proposition 4.2 are disjoint, the angle is in fact at most $\frac{\pi}{m}$. Let $g_{m}$ be the total number of elements counted by these disjoint sets. Consider the case where the potential amalgam is obtained from one relator $w$, of alternating length $m$, i.e., $A(w)=E * F /\langle\langle w\rangle\rangle$.

Question 4.3. If $E, F$ inject into the group $A(w)$ and $\operatorname{order}(A(w)) \geq g_{m}$, is $A \in \Gamma(E, F: m) \Gamma$

Corollary 4.4. (1) If $A \in \Gamma(p, q: 2)$, then $|A| \geq p q$.

(2) If $A \in \Gamma(p, q: 3)$, then $|A| \geq p(p(q-1)+1)$.

(3) If $A \in \Gamma(p, q: 4)$, then $|A| \geq p q(p q-p-q+2)$.

(4) If $A \in \Gamma(p, q: 5)$, then $|A| \geq p\left(p^{2} q^{2}+p^{2}-2 p^{2} q+\right.$ $\left.3 p q-q^{2} p-2 p+1\right)$.

(5) If $A \in \Gamma(p, q: 6)$, then $|A| \geq p q\left(p^{2} q^{2}-2 p^{2} q-\right.$ $\left.2 p q^{2}+p^{2}+q^{2}+5 p q-3 p-3 q+3\right)$.

(6) If $A \in \Gamma(p, 2: m)$, then $|A|>(p-1)^{\lceil m / 2\rceil}$.

The values of $\left(p, q: g_{3}, g_{4}, g_{5}, g_{6}\right)$ for $5 \geq p \geq q \geq 2$ are

$(2,2: 6,8,10,12)$,

$(3,2: 12,18,30,42)$,

$(4,2: 20,32,68,104)$,

$(5,2: 30,50,130,210)$,

$\left(n, 2: n(n+1), 2 n^{2}, n^{3}+n, 2 n\left(n^{2}-n+1\right)\right)$,

$(3,3: 21,45,93,189)$, 
$(4,3: 36,84,228,516)$,

$(5,3: 55,135,455,1095)$,

$(4,4: 52,160,484,1456)$,

$(5,4: 80,260,980,3140)$,

$(5,5: 105,425,1705,6825)$.

Suppose $E$ and $F$ generate the group $A$. Certainly if $E \cap F \neq\{1\}$, when $D=\{1\}$, then the angle is $\pi$.

Remark. Whenever $E \cap N_{A}(F) \neq\{1\}$ there is a relation $x y x^{-1}=y^{\prime}$ with $x \in E$ and $y, y^{\prime} \in F$ so that the angle is at least $\frac{\pi}{2}$. Thus, if $F$ is normal in $A$, the angle is at least $\frac{\pi}{2}$.

\section{Geometric Types: Spherical and Toroidal}

We investigate the possible maps (cf. [Coxeter and Moser 1980]) that support the link of a vertex in a triangle of groups. If the triangle of finite groups has a vertex link, which is a graph having a finite cover that is planar, then we might hope that there is a finite index subgroup of the triangle group that can be thickened up to a three manifold group. A map is a decomposition of a surface $X$ without boundary into $N_{2}$ faces, each of which are disks, as the complement of a graph having $N_{1}$ edges and $N_{0}$ vertices. Given a graph, we shall assume that the map has maximal number of faces or equivalently that the Euler characteristic of the surface is maximal. If the average degree of a vertex is $d$ and the average length of a face circuit is $c$ then the Euler characteristic of a map with this graph is $\chi=N_{0}-N_{1}+N_{2}=\frac{2 N_{1}}{d}-N_{1}+N_{2}=\frac{2 N_{1}}{d}-N_{1}+$ $\frac{2 N_{1}}{c}=2 N_{1}\left(\frac{1}{d}+\frac{1}{c}-\frac{1}{2}\right)$. The Riemann-Hurwitz formula for branched coverings implies that an $r$-fold covering graph lies on a surface $Y$ with $\chi_{Y} \leq r \chi_{X}$. Thus it follows that any graph or finite cover of it can only support a map of nonpositive $\chi$ if

$$
\frac{1}{d}+\frac{1}{c} \leq \frac{1}{2}
$$

If the graph is the link of a vertex in a triangle of groups or any finite cover of it, we know from our previous remarks that

$$
d=\frac{\left(e v_{E}+f v_{F}\right)}{\left(v_{E}+v_{F}\right)}=\frac{2 a}{v_{A}}
$$

and $c \geq 2 \gamma_{A}$, where the angle at vertex $A$ between edge groups $E$ and $F$ is $\frac{\pi}{\gamma}$. Since

$$
\frac{v_{A}}{a}-1=\frac{\left(1-k_{A}\right)}{a}=\frac{1}{e}+\frac{1}{f}-1,
$$

we have

$$
2\left(\frac{1}{d}+\frac{1}{c}-\frac{1}{2}\right) \leq \frac{1}{e}+\frac{1}{f}+\frac{1}{\gamma_{A}}-1
$$

and thus if $\frac{1}{e}+\frac{1}{f}+\frac{1}{\gamma_{A}}<1$ any finite cover can only support a map of negative Euler characteristic. We refer to the vertex group as cospherical if there is a finite cover of the coset graph at the vertex that is a spherical graph. We shall say a triangle of groups is spherical if all the vertex groups are cospherical. If a graph or finite cover of it supports a map on the torus but no map on the sphere then the vertex is called cotoroidal. Certainly the condition $\frac{1}{c}+\frac{1}{d}=\frac{1}{2}$ is necessary. We shall say a triangle of groups is toroidal if the vertex groups are spherical or toroidal, but not all spherical. If the vertex groups are cotoroidal or cospherical, then the link is called geometric type. The triangle of groups is geometric type if all of its vertex groups are of geometric type. The condition

$$
\frac{1}{e}+\frac{1}{f}+\frac{1}{\gamma_{A}} \geq 1
$$

must be satisfied at each vertex for the triangle of groups to be geometric type. Adding these equations we obtain the restriction

$$
\frac{1}{a}+\frac{1}{b}+\frac{1}{c} \geq 2 \chi(\Gamma(\mathcal{T}))-\kappa_{\mathcal{T}}
$$

Of course, this inequality is satisfied if the triangle of groups is Platonic. The solutions to the geometric inequality (4-1) for $e \geq f$ are given in Table 1 .

We begin the classification of geometric Platonic groups.

Lemma 4.5. $\Gamma(2,2: m)=\left\{D_{m}\right\}$.

Proof. Suppose that $A$ is a finite amalgam quotient of $Z_{2} * Z_{2}$, injective on the factors, with shortest 


\begin{tabular}{|r|l|}
\hline$\gamma_{A}$ & \multicolumn{1}{|c|}{$(e, f)$} \\
\hline 2 & $\{(2,2),(3,3),(3,2),(4,4),(4,3),(4,2)$, \\
& $(5,3),(5,2),(6,3),(6,2),(n, 2)$ for $n \geq 7\}$ \\
3 & $\{(2,2),(3,3),(3,2),(4,2),(5,2),(6,2)\}$ \\
4 & $\{(2,2),(3,2),(4,2)\}$ \\
5 & $\{(2,2),(3,2)\}$ \\
6 & $\{(2,2),(3,2)\}$ \\
$\geq 7$ & $\{(2,2)\}$ \\
\hline
\end{tabular}

TABLE 1. Solutions to inequality (4-1) with $e \geq f$.

relator $(x y)^{m}$, where $x$ and $y$ are the generators of the factors. Any other relator is $(x y)^{n}$ and by the Euclidean algorithm $m$ divindes $n$. Thus the group is the dihedral group of order $2 m$ having the presentation

$$
D_{m}=\left\langle x, y \mid x^{2}, y^{2},(x y)^{m}\right\rangle .
$$

(The strings on the right represent relations $x^{2}=1$, $y^{2}=1,(x y)^{m}=1$.)

The dihedral group also has the presentation

$$
D_{m}=\left\langle x, y \mid x^{2}, y^{m}, x y x y\right\rangle .
$$

Lemma 4.6. For $p$ an odd prime and $r \geq 1$, we have $\Gamma\left(Z_{p^{r}}, Z_{2}: 2\right)=\left\{Z_{2 p^{r}}, D_{p^{r}}\right\}$.

Proof. Suppose that $A$ is a finite amalgam quotient of $Z_{2} * Z_{p}^{r}$ injective on the factors, and $x, y$ are the respective generators of each factor. If the shortest relator is of length 4 , it is $x y^{n} x y^{m}$ for some $n, m$. Since the second factor is of prime power order we may replace the generator $y$ by $y^{k}$ for $k$ relatively prime to $p$ and may assume then that the relation is $x y^{p^{i}} x y^{-p^{j} m}$, for $m$ relatively prime to $p$. By this relation, $y^{p^{i}}$ and $y^{-p^{j} m}$ have the same order and thus $i=j$. Now

$$
y^{p^{i}}=x x y^{p^{i}} x x=x y^{p^{i} m} x=y^{p^{2 i} m^{2}},
$$

and thus $p^{i} m^{2}=1 \bmod p^{n-i}$. But this is impossible unless $i=0$; hence we assume $i=0$. Since the group of units mod $p^{r}$ is cyclic there are just two solutions $m= \pm 1$. Hence we obtain just two possible relations, $x y x y$ or $x y x y^{-1}$. This gives the dihedral group and a cyclic group.

Lemma 4.7. (i) $\Gamma(3,3: 2)=\left\{A_{4}, Z_{3} \times Z_{3}\right\}$.

(ii) $\Gamma(3,2: 3)=\left\{A_{4}\right\}$.

(iii) $\Gamma(3,2: 4)=\left\{S_{4}, \mathrm{Cu}_{18}, \mathrm{Cu}_{24}\right\}$.

Proof. In the first case we suppose that $A$ is a finite quotient of $Z_{3} * Z_{3}$, injective on the factors, and $x, y$ are the generators of the factors. The possible relations of length 4, after changing $x$ to $x^{-1}$ or $y$ to $y^{-1}$ if necessary, or possibly switching the roles of $x$ and $y$, are $x y x y, x y x^{-1} y^{-1}$ or $x y x y^{-1}$. For the last relation we see that $y^{2}$ commutes with $x$ and hence $y$ commutes with $x$ and therefore from this relation $x$ maps trivially; this contradicts the faithfulness of each of the factors. The other two relations give the respective groups, either $Z_{3} \times Z_{3}$ for the second relation or for the first relation,

$$
A_{4}=\left\langle x, y \mid x^{3}, y^{3},(x y)^{2}\right\rangle .
$$

They have no quotients for which the factors inject.

In the other cases we suppose that $A$ is a finite quotient of $Z_{2} * Z_{3}$, injective on the factors, and $x, y$ are the generators of the factors. The possible relations after changing $y$ to $y^{-1}$ if necessary are: of length 6 , xyxyxy or $x y x y x y^{-1}$; of length 8, , xyxyxyxy, xyxyxy $x y^{-1}, x y x y x y^{-1} x y^{-1}$, $x y x y^{-1} x y x y^{-1}$. Certainly we have

$$
\begin{aligned}
& A_{4}=\left\langle x, y \mid x^{2}, y^{3},(x y)^{3}\right\rangle, \\
& S_{4}=\left\langle x, y \mid x^{2}, y^{3},(x y)^{4}\right\rangle .
\end{aligned}
$$

The only relations that give presentations of finite groups with injective factors are

$$
\mathrm{Cu}_{18}=\left\langle x, y \mid x^{2}, y^{3},(x y)^{2}\left(x y^{-1}\right)^{2}\right\rangle
$$

of order 18 and

$$
\mathrm{Cu}_{24}=\left\langle x, y \mid x^{2}, y^{3},\left(x y x y^{-1}\right)^{2}\right\rangle
$$

of order 24. Both of these groups abelianize to $Z_{6}$. In this group of order 18, the element $(x y)^{2}=(y x)^{2}$ is central of order 3 with quotient $D_{3}$. The group $\mathrm{Cu}_{24}$ has a center of order 2 generated by $(x y)^{3}$ and having quotient $A_{4}$. It is easy to see that the 
group $\mathrm{Cu}_{18}$ is isomorphic to $D_{3} \times Z_{3}$ (cf. Lemma 6.8 below) and that $\mathrm{Cu}_{24}$ is isomorphic to $Z_{2} \times A_{4}$ (cf. Proposition 4.10 below).

We can refine Proposition 4.1 to handle some other cases.

Proposition 4.8. (i) $1+k_{A} \geq v_{A} / \gamma_{A}$, except if $2=$ $\gamma_{A}=f$ and $e=4$.

(ii) $1+k_{A} \geq a / \gamma_{A}$, except if $\gamma_{A}=2=f$ and $e$ is composite, or if $\gamma_{A}=2$ and $3=f<e$, or if $\gamma_{A}=3, f=2$, and $4 \leq e \leq 5$.

Proof. Since $|E|,|F| \geq 2$, we have $a=\left(e\left(v_{E}\right)+\right.$ $\left.f\left(v_{F}\right)\right) / 2 \geq v_{A}$, so the second inequality is harder to satisfy. We assume that $f \leq e$. For the first inequality we have $\left(1+k_{A}\right) / v_{A}=\left(a / v_{A}\right)+\left(2 / v_{A}\right)-$ 1 and $a=\left(e\left(v_{E}\right)+f\left(v_{F}\right)\right) / 2$, so that $\left(1+k_{A}\right) / v_{A}=$ $\left(e\left(v_{E}\right)+f\left(v_{F}\right)\right) /\left(2\left(v_{E}+v_{F}\right)\right)+2 / v_{A}-1$. Hence if $e, f \geq 3$,

$$
\frac{1+k_{A}}{v_{A}} \geq \frac{3\left(v_{E}+v_{F}\right)}{2\left(v_{E}+v_{F}\right)}+\frac{2}{v_{A}}-1>\frac{1}{2},
$$

so that the inequality is valid for $\gamma_{A} \geq 2$. If $f=2$ and $e \geq 3$ then $a=2\left(v_{F}\right)=e\left(v_{E}\right), v_{A}=(e / 2+1) \times$ $v_{F}$ and therefore, for $e \geq 6$,

$$
\frac{1+k_{A}}{v_{A}} \geq \frac{e-2}{e+2} \geq \frac{1}{2} \geq \frac{1}{\gamma_{A}} .
$$

If $f=2$ and $\gamma_{A} \geq 3$, the first inequality is also valid for $e \geq 2$.

If $f=2, \gamma=2$ and $e$ is an odd prime then $a=2 p$ by Lemma 4.6 ; hence the second inequality is satisfied. If $f=e=3$ and $\gamma=2$ then either $a=9$ or $a=12$ by Lemma 4.7, and in both cases the second inequality is satisfied. There remains to consider the case of $f=2, e=3, \gamma=3$; by Lemma 4.7, we have $a=12$ and the second inequality is valid. This also settles the unresolved cases of the first inequality.

Lemma 4.9. (i) $\Gamma(5,3: 2)=\left\{Z_{15}, A_{5}\right\}$.

(ii) $\Gamma(5,2: 3)=\left\{A_{5}\right\}$.

(iii) $\Gamma(3,2: 5)=\left\{A_{5}\right\}$.

Proof. In the first case we suppose that $A$ is a finite quotient of $Z_{3} * Z_{5}$, injective on the factors, and $x, y$ are the generators of the factors. The possible relations of length 4 are $x y x y^{i}$ or $x y x^{-1} y^{i}$. In the latter case, it follows that $i^{3}=-1 \bmod 5$ and hence $i=-1$ so that the group is $Z_{15}$. In the first case we find by a coset enumeration that if $i=2$ or $i=3$ the group is trivial, and if $i=4$ the group is of order 5. The only appropriate case then is $i=1$; hence we obtain

$$
A_{5}=\left\langle x, y \mid x^{3}, y^{5},(x y)^{2}\right\rangle .
$$

In case (ii) we suppose that $A$ is a finite quotient of $Z_{2} * Z_{5}$, injective on the factors, and $x, y$ are the generators of the factors. The possible relations to consider of length 6 are

$$
\left\{x y x y^{2} x y^{3}, x y x y^{2} x y^{4}, x y x y x y^{i} \text { for } i=1, \ldots, 4\right\} \text {. }
$$

The only nontrivial quotient groups are for the relations $(x y)^{3}$ or $x y x y x y^{3}$. The latter is a group of order 5 . Thus we obtain only the alternating group

$$
A_{5}=\left\langle x, y \mid x^{2}, y^{5},(x y)^{3}\right\rangle .
$$

In case (iii) we suppose that $A$ is a finite quotient of $Z_{2} * Z_{3}$, injective on the factors, and $x, y$ are the generators of the factors. The possible relations to consider after possibly changing $y$ to $y^{-1}$ of length 10 are $(x y)^{5},(x y)^{4} x y^{-1},(x y)^{3}\left(x y^{-} 1\right)^{2}$, $\left(x y x y^{-1}\right)^{2} x y$. The quotients by these relations have orders $60,3,1,1$, respectively. Thus, we obtain only the alternating group

$$
A_{5}=\left\langle x, y \mid x^{2}, y^{3},(x y)^{5}\right\rangle .
$$

Proposition 4.10. (i) In any finite amalgam of

(a) $D_{3}{ }_{Z_{2}} Z_{4}$,

(b) $D_{3} \underset{Z_{2}}{*} D_{2}\left(\cong \mathrm{PGL}_{2}(Z)\right)$,

(c) $Z_{6} \underset{Z_{2}}{*} D_{2}\left(\cong Z_{2} \times \mathrm{PSL}_{2}(Z)\right)$, or

(d) $Z_{6} \underset{Z_{2}}{*} Z_{4} \cong \mathrm{SL}_{2}(Z)$

of order greater than 12 , the image of the unique subgroup of order 3 in the first factor is not normal.

(ii) The finite amalgams of order 24 are, respectively,
(a) none,
(b) $\mathrm{PGL}_{2}\left(Z_{3}\right)\left(\cong S_{4}\right)$, 
(c) $Z_{2} \times \operatorname{PSL}_{2}\left(Z_{3}\right)\left(\cong \mathrm{Cu}_{24}\right)$,

(d) $\mathrm{SL}_{2}\left(Z_{3}\right)$ (isomorphic to the universal central extension $\left.A_{4}\right)$.

Proof. For the first part we use the fact that the subgroup of order three is the unique subgroup of either $D_{3}$ or $Z_{6}$. Suppose that this element of order three is $x$ and that the element generating the second factor (modulo $Z_{2}$ ) is $y$. Denote by $z$ the common element of order two that together with $x$ generates the first factor of order 6 . In case (a),

$$
D_{3} \underset{Z_{2}}{\underset{*}{*}} Z_{4}=\left\langle x, y, z \mid x^{3}, y^{4}, z y^{-2},(z x)^{2}\right\rangle,
$$

and if $y$ centralizes $x$ then finite quotient has order less than 12 ; morever, if $y x y^{-1}=x^{-1}$ the finite amalgam is of order 12 . In case (b),

$$
D_{3} \underset{Z_{2}}{\stackrel{*}{*}} D_{2}=\left\langle x, y, z \mid x^{3}, y^{2}, z^{2},(y z)^{2},(z x)^{2}\right\rangle,
$$

so that if $y$ centralizes $x$ then $y$ commutes with the group $\langle x, z\rangle$ and the amalgam is of order 12 . Otherwise, if $(y x)^{2}=1$ then the group is also of order 12 . In case $(\mathrm{c})$,

$$
Z_{6} \underset{Z_{2}}{\stackrel{*}{ }} D_{2}=\left\langle x, y, z \mid x^{3}, y^{2}, z^{2},(y z)^{2}, x z x^{-1} z\right\rangle,
$$

so that if $y$ centralizes $x$ then the group is a direct product and of order 12; otherwise the quotient is

$$
\left\langle x, y, z \mid x^{3}, y^{2}, z^{2},(y z)^{2},(y x)^{2}, x z x^{-1} z\right\rangle,
$$

also of order 12 . In case $(\mathrm{d})$,

$$
Z_{6} \underset{Z_{2}}{*} Z_{4}=\left\langle x, y, z \mid x^{3}, y^{4}, z y^{-2}, z x z x^{-1}\right\rangle ;
$$

if $y$ centralizes $x$ then the group is a direct product of $\langle x\rangle$ and $\langle y\rangle$; otherwise if $y x y^{-1}=x$ the group is the semidirect product $\left\langle x, y \mid x^{3}, y^{4}, y x y^{-1} x^{-1}\right\rangle$ of order 12 .

Now, to analyze a finite amalgam quotient $G$ of order 24 , it follows from part (i) that there are 4 Sylow 3 -subgroups and thus by conjugation we obtain a homomorphism $G \rightarrow S_{4}$, with kernel $K$ equal to the intersection of the normalizers of the Sylow 3 -subgroups. Since the normalizers $N_{1}, N_{2}$ of two different Sylow-subgroups have order six their common intersection $N_{1} \cap N_{2}$ has order at most 2 . Hence either $K=\{1\}$ and $G \cong S_{4}$ or there is a central subgroup $K$ of order 2 with $G / K \cong A_{4}$.
In the latter case the Sylow 2-subgroup is normal, since it covers the Sylow 2-subgroup of $A_{4}$, and the group is not a direct product by (i); thus the Sylow 2-subgroup has an automorphism of order 3. This situation occurs only in case the Sylow 2-subgroup is $Z_{2}^{3}$ or $Q_{8}$, the quaternions. In the first case every element has order 2 , while in the second there is an element of order 4 whose square is central.

In case (a), $z=y^{2}$ is central and thus $x^{2}=$ $(z x)^{2}=1$; so there are no amalgams of order 24 .

In case (b), if an amalgam has a normal Sylow 2-subgroup it must have only elements of order 2, since the group contains contains a $D_{2}$. The automorphism of order $3, \alpha$, conjugation by $x$, satisfies $\alpha^{2}=\alpha+1$ and thus we have $x\left(x z x^{-1}\right) x^{-1}=$ $z\left(x z x^{-1}\right)=(z x z) x^{-1}=x^{-2}=x$, which is impossible. Therefore the only amalgam is $S_{4}$.

In case (c), since $S_{4}$ has no elements of order 6 , the amalgam has a central element of order 2 , with quotient $A_{4}$; now $x$ centralizes $z$ and cannot centralize $y$ or else the amalgam is of order 12 ; thus $x y x^{-1}$ is an element of order 2 commuting with $y$, and $\langle x, y\rangle$ has order 12 and is isomorphic to $A_{4}$. The amalgam is a direct product of $\langle z\rangle$ and $\langle x, y\rangle$.

In case (d), again the group is not $S_{4}$ because of the elements of order 6 . Since the Sylow 2subgroup is normal, the finite amalgam is a semidirect product of $Q_{8}$ with an element of order 3; hence the nontrivial central extension of $A_{4}$.

The quotients are then easily described because of the given identifications with subgroups and quotients of $\mathrm{GL}_{2}(Z)$.

Lemma 4.11. The vertex groups in $\Gamma(4,2: 3)$ are determined by

(i) $\Gamma\left(Z_{4}, Z_{2}: 3\right)=\left\{S_{4}, O_{20}\right.$, certain $D_{3} \underset{Z_{2}}{*} Z_{4}$ finite amalgams\},

(ii) $\Gamma\left(D_{2}, Z_{2}: 3\right)=\left\{\right.$ certain $D_{3_{Z_{2}}} D_{2}$ finite amalgams $\}$.

The finite amalgams arising from the free products with amalgamation have order divisible by 12 .

Proof. Suppose that $A$ is a amalgam quotient of $Z_{2} * Z_{4}$ having $x, y$ as the generators of the factors. 
The possible relations of length 6 after changing $y$ to $y^{-1}$ if necessary are: $(x y)^{3},(x y)^{2} x y^{-1},(x y)^{2} x y^{2}$, $x y x y^{-1} x y^{2}, x y\left(x y^{2}\right)^{2},\left(x y^{2}\right)^{3}$. The relations that give finite quotients that are injective on the factors are

$$
S_{4}=\left\langle x, y \mid x^{2}, y^{4},(x y)^{3}\right\rangle,
$$

of order 24 , or

$$
O_{20}=\left\langle x, y \mid x^{2}, y^{4}, x y x y^{-1} x y^{2}\right\rangle,
$$

of order 20 . The last relation yields the free product with amalgamation

$$
D_{3} \underset{Z_{2}}{*} Z_{4}=\left\langle x, y \mid x^{2}, y^{4},\left(x y^{2}\right)^{3}\right\rangle,
$$

so we obtain finite amalgams of it.

For the second part, if $A$ is a quotient of $Z_{2} * D_{2}$ having $x, y, z$ as the generators of the factors. The possible relations of length 6 , after changing independent generators of $D_{2}$ to $y$ and $z$, are $(x y)^{3}$, $(x y)^{2} x z$, xyxzxyz. The latter has order 4 and hence does not give an amalgam. The second quotient after replacing $z$ by $x y x y x$, gives $(x y)^{6}=1$, which cannot be an amalgam with $m=3$ since its order is too small. There remains only the finite amalgams of $\mathrm{PGL}_{2}(Z)$,

$$
D_{3} \underset{Z_{2}}{\underset{*}{*}} D_{2}=\left\langle x, y, z \mid x^{2}, y^{2}, z^{2},(y z)^{2},(x y)^{3}\right\rangle .
$$

By a calculation of the Euler characteristic of these free amalgams, the free kernel has rank $1+$ $\frac{a}{12}$ and thus the order of the finite amalgams is divisible by 12 .

Remark. It is interesting to note in connection with Question 4.3 that the group obtained above from the relation $(x y)^{2} x z$ is an amalgam of the subgroups specified, it is in fact isomorphic to $D_{6}$, but there is the shorter relation $(x z y)^{2}$ of length 4 .

Corollary 4.12. $S_{4}$ is the only group of order 24 in $\Gamma(4,2: 3)$, and it occurs in both $\Gamma\left(Z_{4}, Z_{2}: 3\right)$ and $\Gamma\left(D_{2}, Z_{2}: 3\right)$.

Proof. From Lemma 4.11 it follows that the groups order 24 are either $S_{4}$ or arise as finite amalgams of $D_{3} * Z_{4}$ or $D_{3}{ }_{Z} D_{2}$. By Proposition 4.10, the only amalgam of order 24 is isomorphic to $S_{4}$ occuring as an amalgam of $D_{3} * D_{2}$. In this latter case, we have the presentation in $\Gamma\left(D_{2}, Z_{3}: 2\right)$

$$
S_{4}=\left\langle x, y, z \mid x^{2}, y^{2}, z^{2},(y z)^{2},(x y)^{3},(x z)^{3}\right\rangle .
$$

Lemma 4.13. The groups in $\Gamma(4,2: 2)$ are determined by

(i) $\Gamma\left(Z_{4}, Z_{2}: 2\right)=\left\{2 D_{m}, 2^{\prime} D_{m}\right.$ with $m$ even $\}$,

(ii) $\Gamma\left(D_{2}, Z_{2}: 2\right)=\left\{D_{2 m}, Z_{2} \times D_{m}\right.$ with $\left.m \geq 2\right\}$.

Proof. In the first case suppose that $A$ is a quotient of $Z_{2} * Z_{4}$ having $x, y$ as the generators of the factors. The possible relations of length 4 after changing $y$ to $y^{-1}$ if necessary are $(x y)^{2}, x y x y^{-1}$, $\left(x y^{2}\right)^{2}$. These relations give the groups

$$
D_{4}=\left\langle x, y \mid x^{2}, y^{4},(x y)^{2}\right\rangle,
$$

the abelian group $Z_{2} \times Z_{4}$, and amalgams of $D_{2} Z_{2} Z_{4}$. This last group after factoring out by the central element $y^{2}$ has as its amalgam quotients the dihedral groups $D_{m}$. Using the relation $y x y^{-1}=y^{-1} x y$ repeatedly, we may write the presentation as either

$$
2 D_{r+s}=\left\langle x, y \mid x^{2}, y^{4},\left(x y^{2}\right)^{2},(x y)^{r}\left(x y^{-1}\right)^{s}\right\rangle,
$$

or

$$
2^{\prime} D_{r+s}=\left\langle x, y \mid x^{2}, y^{4},\left(x y^{2}\right)^{2},(x y)^{r}\left(x y^{-1}\right)^{s} y^{2}\right\rangle,
$$

for $r+s=m$. We can see that $2 D_{(r-1)+(s+1)}=$ $2^{\prime} D_{r+s}$, by rewriting the relation in the form

$$
\begin{aligned}
(x y)^{r}\left(x y^{-1}\right)^{s} y^{2} & =(x y)^{r}\left(x y^{-1}\right)^{s} x y^{-2} x \\
& =(x y)^{r}\left(x y^{-1}\right)^{s+1} y^{-1} x ;
\end{aligned}
$$

hence, $(x y)^{r-1}\left(x y^{-1}\right)^{s+1}=1$ if and only if

$$
(x y)^{r}\left(x y^{-1}\right)^{s} y^{2}=1 .
$$

Next observe that if we replace the term $\left(x y^{-1}\right)^{s}$ by $\left(x y^{-1}\right)^{s} y^{4 t}$ for appropriate $t$, then $\left(x y^{-1}\right)^{s}$ equals $(x y)^{s}$ or $(x y)^{s} y^{2}$ depending on the parity of $s$. Thus the presentations become

$$
\begin{aligned}
2 D_{m} & =\left\langle x, y \mid x^{2}, y^{4},\left(x y^{2}\right)^{2},(x y)^{m}\right\rangle, \\
2^{\prime} D_{m} & =\left\langle x, y \mid x^{2}, y^{4},\left(x y^{2}\right)^{2},(x y)^{m} y^{2}\right\rangle .
\end{aligned}
$$

Set $u=x y$, so that $u^{-1}=y u y$ and thus $u^{-m}=$ $(y u y)^{m}=y^{2 m-2} y u^{m} y$, so that if $m$ is odd we get 
$u^{-m} y^{2}=y u^{m} y^{2} y$. For the presentations above this gives $y^{2}=1$, so that it is not an amalgam. For $m$ even we can rewrite the presentation using $x=$ $u y^{-1}$, to get $(u y)^{2}=1,\left(u y^{-1}\right)^{2}$; and thus for $m$ even

$$
\begin{aligned}
2 D_{m} & =\left\langle u, y \mid y^{4},(u y)^{2},\left(u y^{-1}\right)^{2}, u^{m}\right\rangle, \\
2^{\prime} D_{m} & =\left\langle u, y \mid y^{4},(u y)^{2},\left(u y^{-1}\right)^{2}, u^{m} y^{2}\right\rangle .
\end{aligned}
$$

This is a group of order $4 m$ having a central element of order 2 with quotient $D_{m}$.

In case (ii) we consider amalgam quotients of $D_{2} * Z_{2}$. Let $x$ generate the second factor. The possible length 4 relations are $x y x z$ or $(x y)^{2}$, where $y$ and $z$ generate $D_{2}$. For the first relation we obtain a group of order 4 so it is not an amalgam. For the second relation we obtain

$$
D_{2} \underset{Z_{2}}{\underset{*}{*}} D_{2}=\left\langle x, y, z \mid x^{2}, y^{2}, z^{2},(y z)^{2},(x y)^{2}\right\rangle,
$$

which has center generated by $y$ with quotient $Z_{2} *$ $Z_{2}$. The amalgam quotients are then either

$Z_{2} \times D_{m}=\left\langle x, y, z \mid x^{2}, y^{2}, z^{2},(y z)^{2},(x y)^{2},(x z)^{m}\right\rangle$

or

$$
\left\langle x, y, z \mid x^{2}, y^{2}, z^{2},(y z)^{2},(x y)^{2},(x z)^{m} y^{-1}\right\rangle .
$$

Since $y z=(x z)^{m} z=(x z)^{m-1} x, x y=x(x z)^{m}=$ $z(x z)^{m-1}$ we can use the relations $x^{2}=y^{2}=z^{2}=1$ to deduce $(y z)^{2}=(x y)^{2}=1$; thus this presentation simplifies to

$$
D_{2 m}=\left\langle x, z \mid x^{2}, z^{2},(x z)^{2 m}\right\rangle .
$$

Finally, the only groups of order not divisible by 8 in $\Gamma(4,2: 2)$ are in $\Gamma\left(D_{2}, Z_{2}: 2\right)$ and are either $D_{2 m}$ or $Z_{2} \times D_{m}$ for $m$ odd.

Corollary 4.14. (i) The order-8 groups in $\Gamma\left(Z_{4}, Z_{2}: 2\right)$ are $D_{4}$ and $Z_{4} \times Z_{2}$.

(ii) The order-8 groups in $\Gamma\left(D_{2}, Z_{2}: 2\right)$ are $D_{4}$ and $Z_{2} \times D_{2}$.

Proof. This follows easily from the previous Lemma. If $m=2$ the presentations given above simplify to

$$
2^{\prime} D_{m}=\left\langle x, y \mid x^{2}, y^{4},\left(x y^{2}\right)^{2},(x y)^{2} y^{2}\right\rangle,
$$

which is $Z_{4} \times Z_{2}$, or

$$
2 D_{2}=\left\langle x, y \mid x^{2}, y^{4},\left(x y^{2}\right)^{2},(x y)^{2}\right\rangle,
$$

which is $D_{4}$.

Lemma 4.15. The vertex groups in $\Gamma(4,3: 2)$ are determined by

(i) $\Gamma\left(Z_{4}, Z_{3}: 2\right)=\left\{S_{4}, T, Z_{12}\right.$, certain $D_{3} \underset{Z_{2}}{*} Z_{4}$ or $Z_{6} * Z_{4}$ finite amalgams $\}$,

(ii) $\Gamma\left(D_{2}, Z_{3}: 2\right)=\left\{\mathrm{Cu}_{24}\right.$, certain $D_{3} Z_{Z_{2}} D_{2}$ or $Z_{6_{Z_{2}}^{*}} D_{2}$ finite amalgams\}.

Proof. Consider first the amalgam quotients of $Z_{3} *$ $Z_{4}$ having $x, y$ as the generators of the factors. The possible relations of length 4 , after changing $y$ to $y^{-1}$ if necessary, are $(x y)^{2}, x y x y^{-1}, x y x^{-1} y$, $x y x^{-1} y^{-1},\left(x y^{2}\right)^{2}, x y^{2} x^{-1} y^{2}, x y x y^{2}$ and $x y x^{-1} y^{2}$. It is easy to see that the last two relations give the trivial group. The first group is

$$
S_{4}=\left\langle x, y \mid x^{3}, y^{4},(x y)^{2}\right\rangle .
$$

The next group is of order 12:

$$
T=\left\langle x, y \mid x^{3}, y^{4}, x y x y^{-1}\right\rangle .
$$

The next relation gives a group of order 6 so it not an amalgam. From the next relation we get $Z_{12}$.

The next two relations give the free amalgams

$$
\begin{aligned}
& D_{3} \underset{Z_{2}}{\underset{*}{*}} Z_{4}=\left\langle x, y, z \mid x^{3}, y^{4},\left(x y^{2}\right)^{2}\right\rangle, \\
& Z_{6} \underset{Z_{2}}{\stackrel{*}{*}} Z_{4}=\left\langle x, y, z \mid x^{3}, y^{4}, x y^{2} x^{-1} y^{2}\right\rangle .
\end{aligned}
$$

In case (ii) the relations are $(x y)^{2}, x y x^{-1} y, x y x z$, $x y x^{-1} z$, where $y, z$ generate $D_{2}$. The first relation gives the group

$$
D_{3} \underset{Z_{2}}{\underset{*}{*}} D_{2}=\left\langle x, y, z \mid x^{3}, y^{2}, z^{2},(y z)^{2},(x y)^{2}\right\rangle,
$$

so we get finite amalgams. The next relation gives

$$
Z_{6} \underset{Z_{2}}{*} D_{2}=\left\langle x, y, z \mid x^{3}, y^{2}, z^{2},(y z)^{2}, x y x^{-1} y\right\rangle,
$$

so we get central extensions of order 2 of $Z_{3} * Z_{2}$ amalgams. In the other cases we can eliminate $z$ to get

$$
\left\langle x, y \mid x^{3}, y^{2},(x y x)^{2},(y x y x)^{2}\right\rangle
$$


which is $Z_{6}$ and so not an amalgam, since it has no element of order 4 . The last case with $z=x y x^{-1}$ gives

$$
\mathrm{Cu}_{24}=\left\langle x, y \mid x^{3}, y^{2},\left(y x y x^{-1}\right)^{2}\right\rangle .
$$

Corollary 4.16. The groups of order 24 in $\Gamma\left(Z_{4}, Z_{3}: 2\right)$ are $S_{4}$ and $\mathrm{SL}_{2}\left(Z_{3}\right)$. The groups of order 24 in $\Gamma\left(D_{2}, Z_{3}: 2\right)$ are $\mathrm{Cu}_{24}$ and $S_{4} \cong \mathrm{PGL}_{2}\left(Z_{3}\right)$.

Proof. From Proposition 4.10, the groups of order 24 in $\Gamma\left(Z_{4}, Z_{3}: 2\right)$ are either $S_{4}$ or $\mathrm{SL}_{2}\left(Z_{3}\right)$. From Lemma 4.15, that the groups of order 24 in $\Gamma\left(D_{2}, Z_{3}: 2\right)$ are either $S_{4}$ (as presented in Corollary 4.12) or $\mathrm{Cu}_{24}$ respectively, or arise as finite amalgams as in Proposition 4.10. We use the presentation

$$
\mathrm{SL}_{2}\left(Z_{3}\right)=\left\langle x, y \mid x^{3}, y^{4}, x y^{2} x^{-1} y^{2},(x y)^{3}\right\rangle,
$$

and also

$$
\mathrm{Cu}_{24}=\left\langle x, y, z \mid x^{3}, y^{2}, z^{2},(y z)^{2}, z x y^{-1} x^{-1}\right\rangle .
$$

\section{Coplanarity}

Let $G(A: E, F: m)$ denote the coset graph of the vertex $A \in \Gamma(E, F: m)$. Denote the complete bipartite graph on sets of size $m$ and $n$ as $K(m, n)$.

Proposition 4.17. The coset graphs of the groups described in Lemmas 4.5-4.9 and in Corollaries 4.12 and 4.14 are coplanar.

Proof. The graph $G\left(D_{m}: Z_{2}, Z_{2}: m\right)$ is a single circuit having $2 m$ vertices, so it is planar. The graphs $G\left(D_{p^{r}}: Z_{p^{r}}, Z_{2}: 2\right)$ and $G\left(Z_{2 p^{r}}: Z_{p^{r}}, Z_{2}: 2\right)$ are both $K\left(p^{r}, 2\right)$, which can be viewed as an equator of $p^{r}$ vertices connected to a $N$ and $S$ pole on $S^{2}$; hence they are planar.

The graph $G\left(A_{4}: Z_{3}, Z_{3}: 2\right)$ can be viewed as the 8 vertices and 12 edges of a cube.

The graph $G\left(Z_{3} \times Z_{3}: Z_{3}, Z_{3}: 2\right)=K(3,3)$ can be imbedded in $P^{2}$ and hence it has a cover in $S^{2}$. The graph $G\left(A_{4}: Z_{3}, Z_{2}: 3\right)$ is the barycentric subdivision of the 1-skeleton of the dual graph of the faces and edges of a tetrahedron, so is planar.

The graph $G\left(\mathrm{Cu}_{18}: Z_{3}, Z_{2}: 4\right)$ is the barycentric subdivision of $K(3,3)$, so it has a planar cover.
The graph $G\left(S_{4}: Z_{3}, Z_{2}: 4\right)$ is the barycentric subdivision of the 1-skeleton of the barycentric subdivision of dual graph of the faces and edges of an octahedron, so it forms the vertices and edges, with barycenters, of a cube; it is planar.

The graph $G\left(\mathrm{Cu}_{24}: Z_{3}, Z_{2}: 4\right)$ can be viewed as barycentric subdivision of the edges of a cube.

For Lemma 4.9, the graph $G\left(A_{5}: Z_{5}, Z_{3}: 2\right)$ can be viewed on the sphere with an upper and lower hemispherical pentagon joined by 5 edges at the midpoints of their sides. Inside each of the pentagons is a 5-pointed star having ten sides, with points at the midpoints of the surrounding pentagon. Inside each of the stars is a single vertex joined to the 5 non-star vertices. See Figure 1.

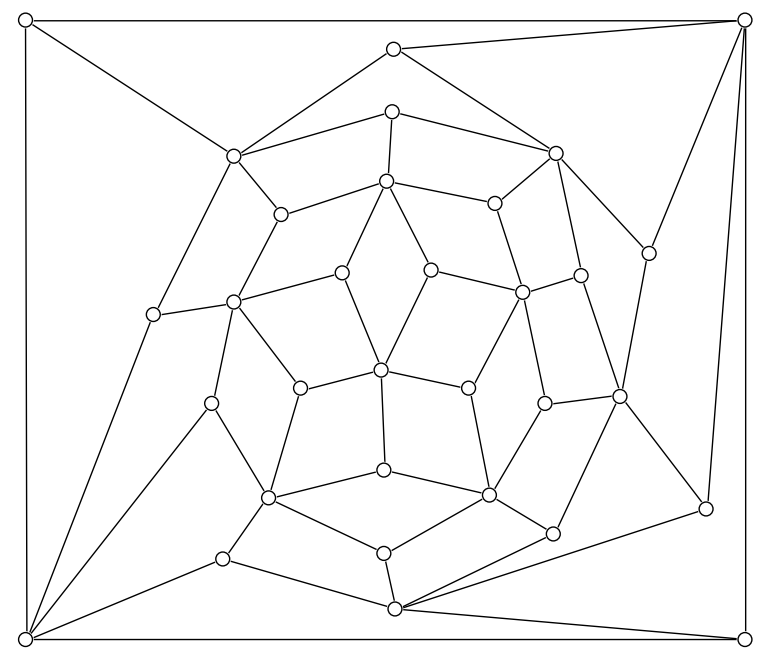

FIGURE 1. $G(60: 5,3: 2)$

The graph $G\left(Z_{15}: Z_{5}, Z_{3}: 2\right)=K(5,3)$ imbeds in a Klein bottle.

The graph $G\left(A_{5}: Z_{5}, Z_{2}: 3\right)$ is the 1-skeleton of the barycentric subdivision of the faces and edges of a dodecahedron, so is the vertices and edges, with barycenters, of an icosahedron; hence it is planar.

The graph $G\left(A_{5}: Z_{3}, Z_{2}: 5\right)$ is the 1-skeleton of the barycentric subdivision of faces and edges of an icosohedron, so it is the vertices and edges, with barycenters, of a dodecahedron; hence it is planar. 
The graphs $G\left(S_{4}: Z_{4}, Z_{2}: 3\right)$ and $G\left(S_{4}: D_{2}, Z_{2}: 3\right)$ occurring in Corollary 4.12 are both obtained from the 1-skeleton of the faces and edges of a cube, so they are the vertices and edges, with barycenters, of an octahedron; hence they are planar.

The graphs of Corollary 4.14 are $K(4,2)$ and can easily be viewed on the sphere as a north and south poles and 4 vertices on the equator joined to the poles.

Proposition 4.18. The coset graphs $G\left(S_{4}: Z_{4}, Z_{3}: 2\right)$ and $G\left(\mathrm{Cu}_{24}: D_{2}, Z_{3}: 2\right)$ in Corollary 4.16 are both planar. The coset graphs of $G\left(S_{4}: D_{2}, Z_{3}: 2\right)$ and $G\left(\mathrm{SL}_{2}\left(Z_{3}\right): Z_{4}, Z_{3}: 2\right)$ are not spherical or toroidal; hence these graphs are not cospherical or cotoroidal.

Proof. The first assertion is justified by Figure 2 .

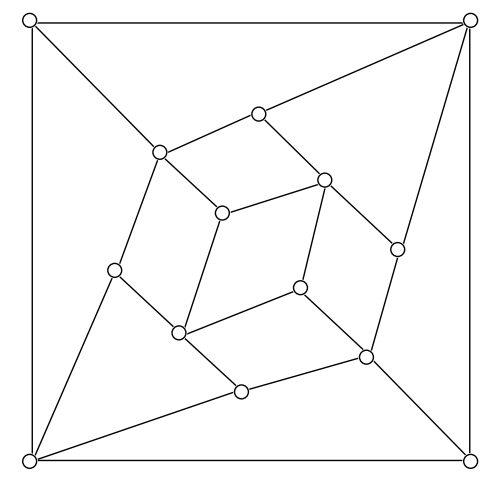

FIGURE 2. $G(24: 4,3: 2)$, planar

In Corollary 4.16, the graphs of $G\left(S_{4}: D_{2}, Z_{3}: 2\right)$ and $G\left(\mathrm{SL}_{2}\left(Z_{3}\right): Z_{4}, Z_{3}: 2\right)$ are the same; they are obtained by gluing two copies of the barycentric subdivision of the 1-skeleton of a tetrahedron at the barycenters. This graph has no imbedding in $P^{2}$ and $S^{2}$ by the solutions to the imbedding problems for $P^{2}$ and $S^{2}$. It also has no torus or Klein bottle imbeddings by observing that a tetrahedron is imbedded uniquely with two faces in an essentally unique way up to the sizes of the polygons and this can not be extended to the other tetrahedron. This graph does have a genus 2 imbedding so it follows from the Riemann-Hurwitz inequality then that any finite cover can not be spherical or toroidal.
Proposition 4.19. The coset graphs of the groups $T$, $Z_{12}$, and $O_{20}$ imbed in $P^{2}$ and thus have a 2-fold planar cover.

Proof. (See Figure 3.) The graphs $G\left(T: Z_{4}, Z_{3}: 2\right)$ and $G\left(Z_{12}: Z_{4}, Z_{3}: 2\right)$ are both $K(4,3)$, and so can be imbedded in $P^{2}$ and have planar covers on $S^{2}$.

The graph $G\left(O_{20}: Z_{4}, Z_{2}: 3\right)$ can be imbedded in a Möbius band with boundary (see Figure 4) and hence in $P^{2}$, so it has a planar cover.

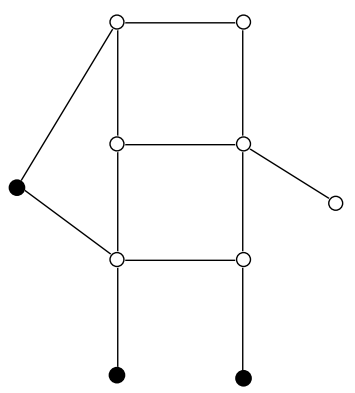

FIGURE 3. $G(12: 4,3: 2)$ on $P^{2}$

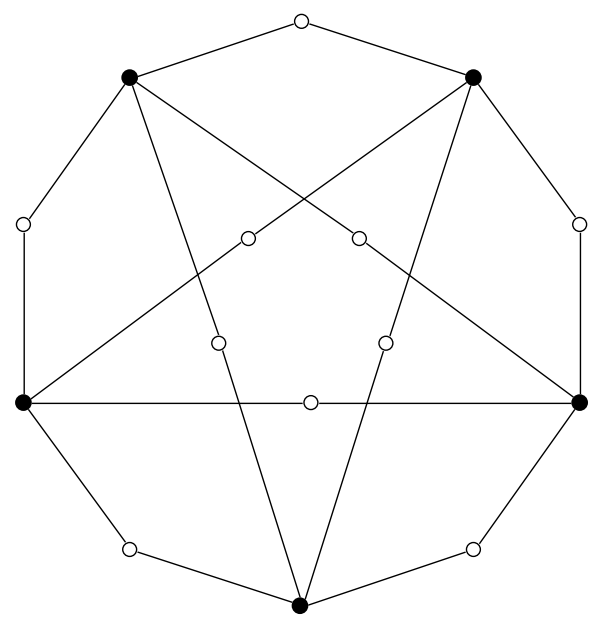

FIGURE 4. $G(20: 4,2: 3)$

\section{NEGATIVELY CURVED GEOMETRIC PLATONIC GROUPS}

In this section we shall classify the data for the class of geometric Platonic triangles of groups. We are also interested in showing that these triangles of finite groups are virtually torsion-free. Certainly, 
this follows if the group has a faithful complex linear representation since it is residually finite and any torsion is conjugate to a vertex stabilizer. An alternate way to show that a triangle of groups $\Gamma$ is virtually torsion-free is to construct enough homomorphisms to finite quotients so that the vertex groups inject into one of the quotients. We are also interested in determining the minimal index of a torsion-free subgroup of finite index. For a triangle of groups there is an obvious surjective homomorphism $\Gamma \rightarrow A_{1}$ obtained by factoring out by the normal subgroup generated by the opposite edge group $G$, where $A_{1}$ is the quotient of $A$ obtained. We call this a collapse. A more general collapse would identify a conjugate of a subgroup of $G$ with a subgroup of $A$. There are also homomorphisms obtained by a folding. A folding is obtained by identification of a subgroup of a conjugate of a subgroup of $E$ with a subgroup of $F$.

Theorem 5.1. (i) Any Platonic group of icosahedral type is negatively curved. There are no geometric negatively curved triangles of icosahedral type.

(ii) A nonpositively curved geometric Platonic group of octahedral type is toroidal and has angles $\frac{\pi}{2}$, $\frac{\pi}{3}, \frac{\pi}{6}$.

(iii) Any geometric negatively curved tetrahedral triangle of groups is one of

$$
\begin{aligned}
& \mathcal{T} h_{1}=\Gamma\left(A_{4}, S_{4}, A_{5}: Z_{3}, Z_{3}, Z_{2}: 2,4,5\right), \\
& \mathcal{T} h_{2}=\Gamma\left(A_{4}, \mathrm{Cu}_{24}, A_{5}: Z_{3}, Z_{3}, Z_{2}: 2,4,5\right), \\
& \mathcal{T} h_{3}=\Gamma\left(A_{4}, A_{5}, A_{5}: Z_{3}, Z_{3}, Z_{2}: 2,5,5\right) .
\end{aligned}
$$

(iv) Any negatively curved dihedral triangle of spherical groups is either an ordinary triangle group or one of these families:

$$
\begin{aligned}
& \mathcal{D} m_{3}^{a}=\Gamma\left(D_{3}, A_{4}, D_{m}: Z_{2}, Z_{3}, Z_{2}: 2,3, m\right), \quad m \geq 7, \\
& \mathcal{D} m_{3}^{b}=\Gamma\left(Z_{6}, A_{4}, D_{m}: Z_{2}, Z_{3}, Z_{2}: 2,3, m\right), \quad m \geq 7, \\
& \mathcal{D} m_{3}^{c}=\Gamma\left(D_{3}, S_{4}, D_{m}: Z_{2}, Z_{3}, Z_{2}: 2,4, m\right), \quad m \geq 5, \\
& \mathcal{D} m_{3}^{d}=\Gamma\left(Z_{6}, S_{4}, D_{m}: Z_{2}, Z_{3}, Z_{2}: 2,4, m\right), \quad m \geq 5 \text {, } \\
& \mathcal{D} m_{3}^{e}=\Gamma\left(D_{3}, \mathrm{Cu}_{24}, D_{m}: Z_{2}, Z_{3}, Z_{2}: 2,4, m\right), \quad m \geq 5, \\
& \mathcal{D} m_{3}^{f}=\Gamma\left(Z_{6}, \mathrm{Cu}_{24}, D_{m}: Z_{2}, Z_{3}, Z_{2}: 2,4, m\right), \quad m \geq 5 \text {, }
\end{aligned}
$$

$$
\begin{array}{lll}
\mathcal{D} m_{3}^{g}=\Gamma\left(D_{3}, A_{5}, D_{m}: Z_{2}, Z_{3}, Z_{2}: 2,5, m\right), & m \geq 4, \\
\mathcal{D} m_{3}^{h}=\Gamma\left(Z_{6}, A_{5}, D_{m}: Z_{2}, Z_{3}, Z_{2}: 2,5, m\right), & & m \geq 4, \\
\mathcal{D} m_{3}^{i}=\Gamma\left(A_{4}, A_{4}, D_{m}: Z_{2}, Z_{3}, Z_{2}: 3,3, m\right), & & m \geq 4, \\
\mathcal{D} m_{3}^{j}=\Gamma\left(A_{4}, S_{4}, D_{m}: Z_{2}, Z_{3}, Z_{2}: 3,4, m\right), & & m \geq 3, \\
\mathcal{D} m_{3}^{k}=\Gamma\left(A_{4}, \mathrm{Cu}_{24}, D_{m}: Z_{2}, Z_{3}, Z_{2}: 3,4, m\right), & & m \geq 3, \\
\mathcal{D} m_{3}^{l}=\Gamma\left(A_{4}, A_{5}, D_{m}: Z_{2}, Z_{3}, Z_{2}: 3,5, m\right), & & m \geq 3, \\
\mathcal{D} m_{3}^{m}=\Gamma\left(S_{4}, S_{4}, D_{m}: Z_{2}, Z_{3}, Z_{2}: 4,4, m\right), & & m \geq 3, \\
\mathcal{D} m_{3}^{n}=\Gamma\left(S_{4}, \mathrm{Cu}_{24}, D_{m}: Z_{2}, Z_{3}, Z_{2}: 4,4, m\right), & & m \geq 3, \\
\mathcal{D} m_{3}^{o}=\Gamma\left(\mathrm{Cu}_{24}, \mathrm{Cu}_{24}, D_{m}: Z_{2}, Z_{3}, Z_{2}: 4,4, m\right), & m \geq 3, \\
\mathcal{D} m_{3}^{p}=\Gamma\left(S_{4}, A_{5}, D_{m}: Z_{2}, Z_{3}, Z_{2}: 4,5, m\right), & & m \geq 3, \\
\mathcal{D} m_{3}^{q}=\Gamma\left(\mathrm{Cu}_{24}, A_{5}, D_{m}: Z_{2}, Z_{3}, Z_{2}: 4,5, m\right), & & m \geq 3, \\
\mathcal{D} m_{3}^{r}=\Gamma\left(A_{5}, A_{5}, D_{m}: Z_{2}, Z_{3}, Z_{2}: 5,5, m\right), & & m \geq 2, \\
\mathcal{D} m_{4}^{a}=\Gamma\left(D_{4}, S_{4}, D_{m}: Z_{2}, Z_{4}, Z_{2}: 2,3, m\right), & & m \geq 7, \\
\mathcal{D} m_{4}^{b}=\Gamma\left(Z_{4} \times Z_{2}, S_{4}, D_{m}: Z_{2}, Z_{4}, Z_{2}: 2,3, m\right), & m \geq 7, \\
\mathcal{D} m_{4}^{c}=\Gamma\left(D_{4}, S_{4}, D_{m}: Z_{2}, D_{2}, Z_{2}: 2,3, m\right), & & m \geq 7, \\
\mathcal{D} m_{4}^{d}=\Gamma\left(D_{2} \times Z_{2}, S_{4}, D_{m}: Z_{2}, D_{2}, Z_{2}: 2,3, m\right), & m \geq 7, \\
\mathcal{D} m_{4}^{e}=\Gamma\left(S_{4}, S_{4}, D_{m}: Z_{2}, Z_{4}, Z_{2}: 3,3, m\right), & & m \geq 4, \\
\mathcal{D} m_{4}^{f}=\Gamma\left(S_{4}, S_{4}, D_{m}: Z_{2}, D_{2}, Z_{2}: 3,3, m\right), & & m \geq 4, \\
\mathcal{D} m_{5}^{a}=\Gamma\left(D_{5}, A_{5}, D_{m}: Z_{2}, Z_{5}, Z_{2}: 2,3, m\right), & & m \geq 7, \\
\mathcal{D} m_{5}^{b}=\Gamma\left(Z_{10}, A_{5}, D_{m}: Z_{2}, Z_{5}, Z_{2}: 2,3, m\right), & & m \geq 7, \\
\mathcal{D} m_{5}^{c}=\Gamma\left(A_{5}, A_{5}, D_{m}: Z_{2}, Z_{5}, Z_{2}: 3,3, m\right), & & m \geq 4 .
\end{array}
$$

Proof. In a cospherical Platonic triangle of groups the vertex groups satisfy

$$
\frac{1}{e}+\frac{1}{f}+\frac{1}{\gamma_{A}}>1
$$

Hence a vertex group is of one of the following types: $\Gamma(2,2: m)$ for $2 \leq m ; \Gamma(3,2: m)$ for $2 \leq$ $m \leq 5 ; \Gamma(4,2: m)$ for $2 \leq m \leq 3 ; \Gamma(5,2: m)$ for $2 \leq$ $m \leq 3 ; \Gamma(n, 2: m)$ for $m=2$ and $n \geq 6 ; \Gamma(3,3: m)$ for $m=2 ; \Gamma(4,3: m)$ for $m=2 ; \Gamma(5,3: m)$ for $m=2$.

Icosahedral type. Since $\frac{1}{a}+\frac{1}{b}+\frac{1}{c} \leq \frac{1}{30}$, any vertex group has order greater than 30. If the triangle is flat, there are two possibilities: all angles are $\frac{\pi}{3}$ but then there is a vertex group in $\Gamma(3,2: 3)$, and its order is too small; or there is a vertex angle of $\frac{\pi}{2}$ and thus there is a vertex group in $\Gamma(5,2: 2)$, $\Gamma(3,2: 2)$, or $\Gamma(5,3: 2)$. In the first two cases the 
order is too small to satisfy the condition above. Hence, the vertex group must be $A_{5}$ and one of the other vertex groups must be in $\Gamma(3,2: 3)$ or $\Gamma(5,2: 3)$. In all of these cases it is imposssible to satisfy Corollary 3.2. Thus also there is no flat icosahedral triangle of finite groups.

Next, if a nonpositively curved Platonic group is of icosahedral type with cospherical or cotoroidal vertices, the angles in $\Gamma\left(5,3: m_{1}\right), \Gamma\left(5,2: m_{2}\right)$, $\Gamma\left(3,2: m_{3}\right)$ are determined by the arguments above or by the restrictions (4-1) on page 197 . We have $\frac{\pi}{m_{1}}=\frac{\pi}{2}$; hence $\frac{\pi}{m_{2}}=\frac{\pi}{3}$; thus there are no solutions for $\frac{\pi}{m_{3}}$, according to the remarks above.

Octahedral type. If a nonpositively curved spherical Platonic group is of octahedral type, then the angles in $\Gamma\left(4,3: m_{1}\right), \Gamma\left(4,2: m_{2}\right), \Gamma\left(3,2: m_{3}\right)$ are by the above restrictions: $\frac{\pi}{m_{1}}=\frac{\pi}{2}$; hence $\frac{\pi}{m_{2}}=\frac{\pi}{3}$; thus there are no solutions for $\frac{\pi}{m_{3}}$ that give a nonpositively curved triangle. If the group is cotoroidal, then $\frac{\pi}{m_{3}}=\frac{\pi}{6}$.

Tetrahedral type. If a negatively curved geometric Platonic group is of tetrahedral type, the restrictions (4-1) imply that the angles in $\Gamma\left(3,3: m_{1}\right)$, $\Gamma\left(3,2: m_{2}\right), \Gamma\left(3,2: m_{3}\right)$ are either $\frac{\pi}{m_{1}}=\frac{\pi}{2}, \frac{\pi}{m_{2}}=\frac{\pi}{4}$, $\frac{\pi}{m_{3}}=\frac{\pi}{5}$ or $\frac{\pi}{m_{1}}=\frac{\pi}{2}, \frac{\pi}{m_{2}}=\frac{\pi}{5}, \frac{\pi}{m_{3}}=\frac{\pi}{5}$. By Corollary 3.2, this gives $\frac{1}{a}+\frac{1}{b}+\frac{1}{c}=\frac{17}{120}$ or $\frac{7}{60}$. However, $a=9$ or $a=12$ and $b=18$ or $b=24$ by Lemma 4.7 , in case $m_{2}=4$ or $b=60$ by Lemma 4.9 in case $m_{2}=5$. The only solutions have $a=12$, $b=24, c=60$ or $a=12, b=60, c=60$ and are given by the families described above.

Dihedral type. If a negatively curved geometric Platonic group is of dihedral type, (4-1) implies that the angles in $\Gamma\left(n, 2: m_{1}\right), \Gamma\left(n, 2: m_{2}\right), \Gamma\left(2,2: m_{3}\right)$ are $\frac{\pi}{m_{1}}=\frac{\pi}{m_{2}}=\frac{\pi}{2}$ in case $n \geq 6$, so not negatively curved.

The equation from Corollary 3.2 now yields

$$
\frac{1}{a}+\frac{1}{b}=\frac{1}{n}+\frac{1}{2 m_{1}}+\frac{1}{2 m_{2}}-\frac{1}{2}
$$

If $n=5$, the possible values of the right-hand side of this equation are $\frac{1}{30}$ (for $m_{1}=2$ and $m_{2}=$ 3 ) and $\frac{7}{60}$ (for $m_{1}=m_{2}=3$ ); while by Lemmas 4.6 and 4.9, the values of the left-hand side are $a=10$, $b=60$ and $a=b=60$, respectively. Both of these give negatively curved groups in the $\mathcal{D} m_{5}$ family.

If $n=4$ then the possible values of the righthand side are $\frac{1}{6}$ (for $m_{1}=2$ and $m_{2}=3$ ) and $\frac{1}{12}$ (for $m_{1}=m_{2}=3$ ); while by Corollary 4.4, the values of the left-hand side are $a \geq 8, b \geq 20$ and $a, b \geq 20$, respectively. Each of these possibilities gives solutions using Corollaries 4.12 and 4.14 for the $\mathcal{D} m_{4}$ family. The solution $a=20$, for example, does not yield a value of $b$ divisible by 12 .

If $n=3$, Lemmas 4.6, 4.7 and 4.9 and Corollary 4.4 give the following facts: $A \in \Gamma(3,2: 2)$ has order $6, B \in \Gamma(3,2 ; 3)$ has order $12 ; A \in \Gamma(3,2: 2)$ has order $6, B \in \Gamma(3,2 ; 4)$ has order 18 or 24 ; $A \in \Gamma(3,2: 2)$ has order $6, B \in \Gamma(3,2 ; 5)$ has order $60 ; A \in \Gamma(3,2: 3)$ has order $12, B \in \Gamma(3,2 ; 3)$ has order 12; $A \in \Gamma(3,2: 3)$ has order $12, B \in \Gamma(3,2 ; 4)$ has order 18 or $24 ; A \in \Gamma(3,2: 3)$ has order 12 , $B \in \Gamma(3,2 ; 5)$ has order $60 ; A \in \Gamma(3,2: 4)$ has order 18 or $24, B \in \Gamma(3,2 ; 4)$ has order 18 or 24 ; $A \in \Gamma(3,2: 4)$ has order 18 or $24, B \in \Gamma(3,2 ; 5)$ has order $60 ; A \in \Gamma(3,2: 5)$ has order $60, B \in \Gamma(3,2 ; 5)$ has order 60 . Meanwhile, the right-hand side of $(5-1)$ is $\frac{1}{4}, \frac{5}{24}, \frac{11}{60}, \frac{1}{6}, \frac{1}{8}, \frac{1}{10}, \frac{1}{12}, \frac{7}{120}, \frac{1}{30}$. Using the previous corollaries we obtain all the group data of the family $\mathcal{D} m_{3}$.

It is straightforward to obtain presentations for these groups. In all cases the groups are uniquely determined from the data. Some of the groups can be described as Coxeter groups or their rotation subgroups. A Coxeter group with reflection generators $R_{1}, R_{2}, R_{3}, R_{4}$ and products of orders $m_{i j}$ has a subgroup of index two that is its rotation subgroup. This subgroup is generated by $a=R_{2} R_{1}$, $b=R_{4} R_{1}, c=R_{3} R_{1}$ having relations

$$
\begin{aligned}
a^{m_{12}} & =b^{m_{14}}=c^{m_{13}}=\left(b a^{-1}\right)^{m_{24}} \\
& =\left(c a^{-1}\right)^{m_{23}}=\left(b c^{-1}\right)^{m_{34}}=1 .
\end{aligned}
$$

The following groups are rotation subgroups of Coxeter groups or full Coxeter groups (the latter 
are marked with $\dagger$ ). Hence they are residually finite and virtually torsion-free:

$$
\begin{aligned}
& \mathcal{T} h_{1}=\left\langle x, y, z \mid x^{3}, y^{3}, z^{2},(x y)^{2},(x z)^{4},(y z)^{5}\right\rangle, \\
& \mathcal{T} h_{3}=\left\langle x, y, z \mid x^{3}, y^{3}, z^{2},(x y)^{2},(x z)^{5},(y z)^{5}\right\rangle, \\
& \mathcal{D} m_{3}^{a}=\left\langle x, y, z \mid x^{2}, y^{2}, z^{3},(x y)^{m},(x z)^{2},(y z)^{3}\right\rangle, \\
& \mathcal{D} m_{3}^{c}=\left\langle x, y, z \mid x^{2}, y^{2}, z^{3},(x y)^{m},(x z)^{2},(y z)^{4}\right\rangle, \\
& \mathcal{D} m_{3}^{g}=\left\langle x, y, z \mid x^{2}, y^{2}, z^{3},(x y)^{m},(x z)^{2},(y z)^{5}\right\rangle, \\
& \mathcal{D} m_{3}^{i}=\left\langle x, y, z \mid x^{2}, y^{2}, z^{3},(x y)^{m},(x z)^{3},(y z)^{3}\right\rangle, \\
& \mathcal{D} m_{3}^{j}=\left\langle x, y, z \mid x^{2}, y^{2}, z^{3},(x y)^{m},(x z)^{3},(y z)^{4}\right\rangle, \\
& \mathcal{D} m_{3}^{l}=\left\langle x, y, z \mid x^{2}, y^{2}, z^{3},(x y)^{m},(x z)^{3},(y z)^{5}\right\rangle, \\
& \mathcal{D} m_{3}^{m}=\left\langle x, y, z \mid x^{2}, y^{2}, z^{3},(x y)^{m},(x z)^{4},(y z)^{4}\right\rangle, \\
& \mathcal{D} m_{3}^{p}=\left\langle x, y, z \mid x^{2}, y^{2}, z^{3},(x y)^{m},(x z)^{4},(y z)^{5}\right\rangle, \\
& \mathcal{D} m_{3}^{r}=\left\langle x, y, z \mid x^{2}, y^{2}, z^{3},(x y)^{m},(x z)^{5},(y z)^{5}\right\rangle, \\
& \mathcal{D} m_{4}^{a}=\left\langle x, y, z \mid x^{2}, y^{2}, z^{4},(x y)^{m},(x z)^{2},(y z)^{3}\right\rangle ; \\
& \dagger \mathcal{D} m_{4}^{d}=\langle x, y, z| x^{2}, y^{2}, z^{2}, w^{2},(z w)^{2} \text {, } \\
& \left.(x y)^{m},(y z)^{3},(y w)^{3},(x z)^{2},(x w)^{4}\right\rangle, \\
& \mathcal{D} m_{4}^{e}=\left\langle x, y, z \mid x^{2}, y^{2}, z^{4},(x y)^{m},(x z)^{3},(y z)^{3}\right\rangle, \\
& \dagger \mathcal{D} m_{4}^{f}=\langle x, y, z| x^{2}, y^{2}, z^{2}, w^{2},(z w)^{2} \text {, } \\
& \left.(x y)^{m},(y z)^{3},(y w)^{3},(x z)^{3},(x w)^{3}\right\rangle, \\
& \mathcal{D} m_{5}^{a}=\left\langle x, y, z \mid x^{2}, y^{2}, z^{5},(x y)^{m},(x z)^{2},(y z)^{3}\right\rangle, \\
& \mathcal{D} m_{5}^{c}=\left\langle x, y, z \mid x^{2}, y^{2}, z^{5},(x y)^{m},(x z)^{3},(y z)^{3}\right\rangle \text {. } \\
& \mathcal{T} h_{2}=\left\langle x, y, z \mid x^{3}, y^{3}, z^{2},(x y)^{2},(x z)^{5},\left(z y z y^{-1}\right)^{2}\right\rangle, \\
& \mathcal{D} m_{3}^{b}=\left\langle x, y, z \mid x^{2}, y^{2}, z^{3},(x y)^{m},(y z)^{3}, x z x^{-1} z^{-1}\right\rangle, \\
& \mathcal{D} m_{3}^{d}=\left\langle x, y, z \mid x^{2}, y^{2}, z^{3},(x y)^{m},(y z)^{4}, x z x^{-1} z^{-1}\right\rangle, \\
& \mathcal{D} m_{3}^{e}=\left\langle x, y, z \mid x^{2}, y^{2}, z^{3},(x y)^{m},(x z)^{2},\left(y z y z^{-1}\right)^{2}\right\rangle, \\
& \mathcal{D} m_{3}^{f}=\left\langle x, y, z \mid x^{2}, y^{2}, z^{3},(x y)^{m},\left(y z y z^{-1}\right)^{2}, x z x^{-1} z^{-1}\right\rangle, \\
& \mathcal{D} m_{3}^{h}=\left\langle x, y, z \mid x^{2}, y^{2}, z^{3},(x y)^{m},(y z)^{5}, x z x^{-1} z^{-1}\right\rangle \text {, } \\
& \mathcal{D} m_{3}^{k}=\left\langle x, y, z \mid x^{2}, y^{2}, z^{3},(x y)^{m},(x z)^{3},\left(y z y z^{-1}\right)^{2}\right\rangle, \\
& \mathcal{D} m_{3}^{n}=\left\langle x, y, z \mid x^{2}, y^{2}, z^{3},(x y)^{m},(x z)^{4},\left(y z y z^{-1}\right)^{2}\right\rangle, \\
& \mathcal{D} m_{3}^{o}=\left\langle x, y, z \mid x^{2}, y^{2}, z^{3},(x y)^{m},\left(x z x z^{-1}\right)^{2},\left(y z y z^{-1}\right)^{2}\right\rangle \text {, } \\
& \mathcal{D} m_{3}^{q}=\left\langle x, y, z \mid x^{2}, y^{2}, z^{3},(x y)^{m},(y z)^{5},\left(x z x z^{-1}\right)^{2}\right\rangle, \\
& \mathcal{D} m_{4}^{b}=\left\langle x, y, z \mid x^{2}, y^{2}, z^{4},(x y)^{m},(y z)^{3}, x z x^{-1} z^{-1}\right\rangle,
\end{aligned}
$$

$\mathcal{D} m_{4}^{c}=\langle x, y, z| x^{2}, y^{2}, z^{2}, w^{2},(z w)^{2},(x y)^{m}$, $\left.(y z)^{3},(y w)^{3},(x z)^{2},(x w)^{4} z^{-1}\right\rangle$ $\mathcal{D} m_{5}^{b}=\left\langle x, y, z \mid x^{2}, y^{2}, z^{5},(x y)^{m},(y z)^{3}, x z x^{-1} z^{-1}\right\rangle$.

The groups $\mathcal{D} m_{3}^{d}, \mathcal{D} m_{3}^{e}, \mathcal{D} m_{3}^{f}, \mathcal{D} m_{3}^{n}, \mathcal{D} m_{3}^{o}$ have a collapse onto $D_{m}$ for $z=1$. Furthermore, if $m$ is even we may replace $m$ by 2 and consider the folds or collapses for these groups to get injections of the other two vertex stabilizers. The groups

$$
\begin{aligned}
& \mathcal{D} 2_{3}^{d}=\left\langle x, y, z \mid x^{2}, y^{2}, z^{3},(x y)^{2},(y z)^{4}, x z x^{-1} z^{-1}\right\rangle, \\
& \mathcal{D} 2_{3}^{e}=\left\langle x, y, z \mid x^{2}, y^{2}, z^{3},(x y)^{2},(x z)^{2},\left(y z y z^{-1}\right)^{2}\right\rangle, \\
& \mathcal{D} 2_{3}^{f}=\left\langle x, y, z \mid x^{2}, y^{2}, z^{3},(x y)^{2},\left(y z y z^{-1}\right)^{2}, x z x^{-1} z^{-1}\right\rangle
\end{aligned}
$$

are of order 48 and the vertex stabilizers inject. Folding $x=y$ on

$$
\mathcal{D} 2_{3}^{o}=\left\langle x, y, z \mid x^{2}, y^{2}, z^{3},(x y)^{2},\left(x z x z^{-1}\right)^{2},\left(y z y z^{-1}\right)^{2}\right\rangle
$$

gives an injection of the vertex stabilizers. The group

$$
\mathcal{D} 2_{3}^{n}=\left\langle x, y, z \mid x^{2}, y^{2}, z^{3},(x y)^{2},(x z)^{4},\left(y z y z^{-1}\right)^{2}\right\rangle
$$

has a permutation representation

$$
\begin{aligned}
& x \rightarrow(34)(56), \\
& y \rightarrow(35)(46), \\
& z \rightarrow(123)(578)
\end{aligned}
$$

with image of order 192 that gives an injection of the two vertex stabilizers.

Thus the groups $\mathcal{D} m_{3}^{d}, \mathcal{D} m_{3}^{e}, \mathcal{D} m_{3}^{f}, \mathcal{D} m_{3}^{n}, \mathcal{D} m_{3}^{o}$ are virtually torsion-free for all $m$ even.

The group $\mathcal{T} h_{2}$ has a permutation representation

$$
\begin{aligned}
& x \rightarrow(234)(798), \\
& y \rightarrow(235)(687), \\
& z \rightarrow(12)(36)(57)(810)
\end{aligned}
$$

with image of order 960, which gives an injection of each of the vertex stabilizers and hence it is virtually torsion-free. 


\section{FLAT PLATONIC GROUPS}

\section{Tetrahedral}

Theorem 6.1. The tetrahedral flat Platonic groups having an angle of $\frac{\pi}{2}$ are

$$
\begin{aligned}
& \mathcal{T}_{1}=\Gamma\left(A_{4}, S_{4}, S_{4}: Z_{3}, Z_{3}, Z_{2}: 2,4,4\right), \\
& \mathcal{T}_{2}=\Gamma\left(A_{4}, \mathrm{Cu}_{24}, \mathrm{Cu}_{24}: Z_{3}, Z_{3}, Z_{2}: 2,4,4\right), \\
& \mathcal{T}_{3}=\Gamma\left(A_{4}, S_{4}, \mathrm{Cu}_{24}: Z_{3}, Z_{3}, Z_{2}: 2,4,4\right) .
\end{aligned}
$$

These groups are uniquely determined by the triangle of groups data. They are all spherical and virtually torsion-free.

Proof. In the tetrahedral case the vertex angle $\frac{\pi}{2}$ has edges of orders 3 and 3 , since otherwise by Lemma 4.6 the vertex order would be of order 6 contradicting Corollary 3.2. Hence by Lemma 4.7 the order there is 9 or 12 . In the isosceles cases the other vertices have order 18 or 24 by Lemma 4.7 . Now by Corollary 3.2 and Lemma 4.7, it follows that the only possibilities are $\mathcal{T}_{i}, i=1,2,3$.

If the triangle is not isosceles, there is another vertex group of order 12 by Lemma 4.7. Hence in this case it is impossible to satisfy Corollary 3.2. They are spherical by Proposition 4.17.

The presentations of the groups of Theorem 6.1 are

$\mathcal{T}_{1}=\left\langle x, y, z \mid x^{3}, y^{3}, z^{2},(x y)^{2},(x z)^{4},(y z)^{4}\right\rangle$, $\mathcal{T}_{2}=\left\langle x, y, z \mid x^{3}, y^{3}, z^{2},(x y)^{2},\left(z x z x^{-1}\right)^{2},\left(z y z y^{-1}\right)^{2}\right\rangle$, $\mathcal{T}_{3}=\left\langle x, y, z \mid x^{3}, y^{3}, z^{2},(x y)^{2},(x z)^{4},\left(z y z y^{-1}\right)^{2}\right\rangle$.

In each of these cases, if we change the mapping of an edge to a vertex group, say $x$ to $x^{-1}$, this gives an equivalent presentation so the data determines the groups. In general, we can use the following equivalences to rewrite presentations: $1=(x y)^{k}$ if and only if $1=(y x)^{k}$, and also if $x$ is of order 2 then $1=(x y)^{k}$ if and only if $1=\left(y^{-1} x\right)^{k}$ if and only if $1=\left(x y^{-1}\right)^{k}$.

In the first case the group $\mathcal{T}_{1}$ is in fact the rotation subgroup of index 2 in the Coxeter group with diagram 4 ? The group $\mathcal{T}_{1}$ is a discrete group of isometries in hyperbolic space so it is virtually torsion-free. By a fold of the edge groups of order $3, x=z y z$, we have a surjective homomorphism $\mathcal{T}_{1} \rightarrow S_{4}$, which is injective on all the vertex groups. Thus, the minimal torsion-free index is 24 , since there is a subgroup $S_{4}$.

By a fold of the edge groups, $x^{-1}=z y z$, we have a homomorphism $\mathcal{T}_{2} \rightarrow \mathrm{Cu}_{24}$ that is injective on the vertex groups. Thus, $\mathcal{T}_{2}$ is virtually torsionfree. Also, the minimal torsion-free index is 24 , since there is a subgroup $\mathrm{Cu}_{24}$

To see that $\mathcal{T}_{3}$ is virtually torsion is more complicated. There is a homomorphism $\mathcal{T}_{3} \rightarrow S_{7}$ defined by: $x \rightarrow$ (235)(476), $y \rightarrow(246)(375), z \rightarrow$ (12)(46). The image is the simple group of order 168. It is easy to see that this homomorphism is injective on the vertex groups; hence the kernel of this homomorphism is torsion-free.

\section{Equilateral}

Theorem 6.2. A flat Platonic group with angles $\left[\frac{\pi}{3}, \frac{\pi}{3}, \frac{\pi}{3}\right]$ is of dihedral type and is one of

$$
\begin{aligned}
& \mathcal{E}_{2}=\Gamma\left(D_{3}, D_{3}, D_{3} ; Z_{2}, Z_{2}, Z_{2} ; 3,3,3\right), \\
& \mathcal{E}_{3}=\Gamma\left(D_{3}, A_{4}, A_{4} ; Z_{2}, Z_{2}, Z_{3} ; 3,3,3\right), \\
& \mathcal{E}_{4}=\Gamma\left(D_{3}, S_{4}, S_{4} ; Z_{2}, Z_{2}, Z_{4} ; 3,3,3\right), \\
& \mathcal{E}_{2^{2}}=\Gamma\left(D_{3}, S_{4}, S_{4} ; Z_{2}, Z_{2}, D_{2} ; 3,3,3\right), \\
& \mathcal{E}_{5}=\Gamma\left(D_{3}, A_{5}, A_{5} ; Z_{2}, Z_{2}, Z_{5} ; 3,3,3\right) .
\end{aligned}
$$

These groups are uniquely determined by the triangle of groups data. They are all spherical and virtually torsion-free.

Proof. Now in a triangle with all three angles equal to 3 , there is for dihedral type, a vertex group $A \in$ $\Gamma(2,2: 3)$ that has order 6 , and for the other types there is a vertex group $A \in \Gamma(3,2: 3)$ that has order 12. The latter is impossible in the octahedral type since that order is too large. For the tetrahedral type, we have also $B \in \Gamma(3,2: 3)$ of order 12 , and this gives a sum contradicting Corollary 3.2. Now, for the dihedral type we have

$$
\frac{1}{6}+\frac{1}{b}+\frac{1}{c}=\frac{1}{g}
$$


and hence $g \leq 5$. By Lemma 4.5, if $g=2$ then $B=C=D_{3}$, so we get $\varepsilon_{2}$; by Lemma 4.7 , if $g=3$ then $B=C=A_{4}$, so we obtain $\mathcal{E}_{3}$; if $g=5$ then $B, C \in \Gamma(5,2: 3)$, which by Lemma 4.9 is $A_{5}$, so we obtain $\mathcal{E}_{5}$. Now if $g=4$ then vertex groups $B, C \in \Gamma(4,2: 3)$ and hence by Lemma 4.11 and its Corollary 4.12 we obtain either $\mathcal{E}_{4}$ or $\mathcal{E}_{2^{2}}$ depending on whether or not the edge group of order 4 is cyclic or not. A group $B$ of order 20 is impossible since then $c=30$ and is not divisible by 12 .

The presentations of these groups are

$$
\begin{aligned}
& \mathcal{E}_{2}=\left\langle x, y, z \mid x^{2}, y^{2},(x y)^{3}, z^{2},(x z)^{3},(y z)^{3}\right\rangle, \\
& \mathcal{E}_{3}=\left\langle x, y, z \mid x^{2}, y^{2},(x y)^{3}, z^{3},(x z)^{3},(y z)^{3}\right\rangle, \\
& \mathcal{E}_{4}=\left\langle x, y, z \mid x^{2}, y^{2},(x y)^{3}, z^{4},(x z)^{3},(y z)^{3}\right\rangle, \\
& \mathcal{E}_{2^{2}}=\langle x, y, z, w| x^{2}, y^{2}, z^{2}, w^{2},(z w)^{2}, \\
& \left.\quad(x y)^{3},(x z)^{3},(x w)^{3},(y z)^{3},(y w)^{3}\right\rangle, \\
& \mathcal{E}_{5}=\left\langle x, y, z \mid x^{2}, y^{2},(x y)^{3}, z^{5},(x z)^{3},(y z)^{3}\right\rangle .
\end{aligned}
$$

If we change any edge identifications, say $z$ to $z^{-1}$, this gives an equivalent presentation in cases $\mathcal{E}_{i}$, for $i=3,4,5$. In the case of $\mathcal{E}_{2^{2}}$ the subgroup $D_{2}$ has the automorphism $z \rightarrow z, w \rightarrow w z$. We can easily modify the presentation using a Tietze transformation $t=w z$ to get the equivalent presentation $\mathcal{E}_{2^{2}}=\langle x, y, z, w| x^{2}, y^{2}, z^{2}, w^{2},(z w)^{2},(x y)^{3}$, $\left.(x z)^{3},(x w z)^{3},(y z)^{3},(y w z)^{3}\right\rangle$. Hence, the presentations in all cases are uniquely determined from the data.

The group $\mathcal{E}_{2}$ is a triangle group, hence a Coxeter group with diagram $?$. The groups $\mathcal{E}_{3}, \mathcal{E}_{4}, \mathcal{E}_{5}$ are rotation subgroups of Coxeter groups with the respective diagrams

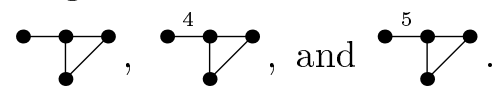

The group $\mathcal{E}_{2^{2}}$ is, in fact, the Coxeter group with the diagram . Thus these are all virtually torsion-free since they are complex linear groups. We can fold $x y=1$ to obtain homomorphisms $\mathcal{E}_{3} \rightarrow A_{4}, \mathcal{E}_{2^{2}} \rightarrow S_{4}$, and $\mathcal{E}_{5} \rightarrow A_{5}$, and also fold $z=x$ to obtain

$$
\mathcal{E}_{4} \rightarrow D_{3} \times S_{4}
$$

which give injections on some of the vertex stabilizers.

In fact, $\mathcal{E}_{3}$ is isomorphic to $\mathrm{PSL}_{2}\left(Z\left[\frac{1}{2}(1+\sqrt{-3})\right]\right)$ [Alperin 1980] and the minimal index torsion-free normal subgroup has index 12 . In the case $\mathcal{E}_{4}$, factoring by the normal subgroup generated by $(x y z)^{2}$ gives a quotient of order 72 injective on all vertex stabilizers.

\section{Dihedral}

Lemma 6.3. The groups of $\Gamma(2 p, 2: 2)$, for odd primes $p$, are

(i) $\Gamma\left(Z_{2 p}, Z_{2}: 2\right)=\left\{D_{2 p}, Z_{2} \times Z_{2 p}\right.$, extensions of a cyclic group of order $p$ by a dihedral group, central extensions of a cyclic group of order 2 by a dihedral group $\}$,

(ii) $\Gamma\left(D_{p}, Z_{2}: 2\right)=\left\{D_{2 p}\right.$, extensions of a cyclic group of order $p$ by a dihedral group $\}$.

Proof. In the first case we consider the possible relations $x y x y, x y x y^{-1}, x y^{2} x y^{2}, x y^{p} x y^{p}$, where $x$ generates the $Z_{2}$ factor and $y$ generates the $Z_{2 p}$ factor. The first relation gives the dihedral group $D_{2 p}$. The second relation gives $Z_{2} \times Z_{2 p}$. The next relation gives amalgams of

$$
D_{p}^{\underset{Z_{p}}{*}} Z_{2 p}=\left\langle x, y, z \mid x^{2}, y^{2 p},\left(x y^{2}\right)^{2}\right\rangle ;
$$

after factoring out by the group generated by $y^{2}$ of order $p$ we get dihedral groups. The last relation gives amalgams of

$$
D_{2} \underset{Z_{2}}{*} Z_{2 p}=\left\langle x, y, z \mid x^{2}, y^{2 p},\left(x y^{p}\right)^{2}\right\rangle ;
$$

after factoring out by the central group generated by $y^{p}$ of order 2 we get dihedral groups.

In the second case, we consider the possible relations $x y x z, x y z x y z$ and $x y z x z y$ where $x$ generates the $Z_{2}$ factor and $y, z$ are elements of order 2 that generate the $D_{p}$ factor. The groups that arise are

$$
D_{2 p}=\left\langle x, y, z \mid x^{2}, y^{2}, z^{2},(y z)^{p}, x y x z\right\rangle ;
$$

amalgams of

$$
D_{p} \underset{Z_{p}}{*} D_{p}=\left\langle x, y, z \mid x^{2}, y^{2}, z^{2},(y z)^{p},(x y z)^{2}\right\rangle,
$$


which are extensions of a cyclic group generated by $y z$ of order $p$ by a dihedral group or amalgams of

$$
Z_{2 p} \underset{Z_{p}}{\underset{*}{*}} D_{p}=\left\langle x, y, z \mid x^{2}, y^{2}, z^{2},(y z)^{p}, x y z x z y\right\rangle,
$$

which are extensions of a cyclic group generated by $y z$ of order $p$ by a dihedral group.

Proposition 6.4. A dihedral Platonic group with angles $\left[\frac{\pi}{2}, \frac{\pi}{4}, \frac{\pi}{4}\right]$ has order data given by one of

$$
\begin{aligned}
& \mathcal{R}_{2}=\Gamma(4,8,8 ; 2,2,2 ; 2,4,4), \\
& \mathcal{R}_{3}=\Gamma(4,24,24 ; 2,2,3 ; 2,4,4), \\
& \mathcal{R}_{4}=\Gamma(24,6,8 ; 2,3,2 ; 4,2,4), \\
& \mathcal{R}_{6}^{1}=\Gamma\left(72,36, D_{4} ; 2,6,2 ; 4,2,4\right), \\
& \mathcal{R}_{6}^{2}=\Gamma\left(120,30, D_{4} ; 2,6,2: 4,2,4\right) .
\end{aligned}
$$

Proof. A vertex group $A \in \Gamma(2,2: 2)$ is of order 4 , and it follows from Corollary 3.2 that in the dihedral case the other edge group has order $f \leq 3$. If $f=2$, this determines $\mathcal{R}_{2}$. If $f=3$, then the vertex groups are in $\Gamma(3,2: 4)$ and this by Lemma 4.7 gives the family $\mathcal{R}_{3}$.

Otherwise, there is a vertex group $B \in \Gamma(f, 2: 2)$ and a vertex group $C \in \Gamma(2,2: 4)$ of order 8 ; now $a, b$ are divisible by $f$, so $a=a_{1} f, a_{1} \geq 2, b=b_{1} f$, $b_{1} \geq 2$ so that

$$
\frac{1}{a_{1} f}+\frac{1}{b_{1} f}+\frac{1}{8}=\frac{1}{f}
$$

by Corollary 3.2. Therefore

$$
\frac{f}{8}=1-\frac{1}{a_{1}}-\frac{1}{b_{1}},
$$

so that $3 \leq f \leq 7$. It follows from Lemma 4.6 now that $f=5,7$ can not satisfy the equation of Corollary 3.2. For $f=3$ we obtain $C=D_{4}$, either $B=Z_{6}$ or $B=D_{3}$ and either $A=S_{4}$ or $A=\mathrm{Cu}_{24}$. These give the $\mathcal{R}_{4}$ family. If $f=4$ then we have the equation $\frac{1}{2}=\frac{1}{a_{1}}+\frac{1}{b_{1}}$ having solutions in $\left(a_{1}, b_{1}\right) \in\{(4,4),(3,6),(6,3)\}$. By Corollary 4.4, $A \in \Gamma(4,2: 4)$ is of order at least 32 by Corollary 4.4 , and thus this case is impossible. If $f=6$ we have $A \in \Gamma(6,2: 4)$ of order at least 72 ; also, $\frac{1}{4}=\frac{1}{a_{1}}+\frac{1}{b_{1}}$, with $a_{1} \geq 12, b_{1} \geq 5$, so the solutions are $\left(a_{1}, b_{1}\right) \in\{(20,5),(12,6)\}$ giving the potential family $\mathcal{R}_{6}$.

Theorem 6.5. A dihedral Platonic group with angles $\left[\frac{\pi}{2}, \frac{\pi}{4}, \frac{\pi}{4}\right]$ that is of geometric type is spherical and one of the following:

$$
\begin{aligned}
& \mathcal{R}_{2}=\Gamma\left(D_{2}, D_{4}, D_{4} ; Z_{2}, Z_{2}, Z_{2} ; 2,4,4\right), \\
& \mathcal{R}_{3}^{1}=\Gamma\left(D_{2}, S_{4}, S_{4} ; Z_{2}, Z_{2}, Z_{3} ; 2,4,4\right), \\
& \mathcal{R}_{3}^{2}=\Gamma\left(D_{2}, \mathrm{Cu}_{24}, \mathrm{Cu}_{24} ; Z_{2}, Z_{2}, Z_{3} ; 2,4,4\right), \\
& \mathcal{R}_{3}^{3}=\Gamma\left(D_{2}, S_{4}, \mathrm{Cu}_{24} ; Z_{2}, Z_{2}, Z_{3} ; 2,4,4\right), \\
& \mathcal{R}_{4}^{1}=\Gamma\left(S_{4}, D_{3}, D_{4} ; Z_{2}, Z_{3}, Z_{2} ; 4,2,4\right), \\
& \mathcal{R}_{4}^{2}=\Gamma\left(\mathrm{Cu}_{24}, D_{3}, D_{4} ; Z_{2}, Z_{3}, Z_{2} ; 4,2,4\right), \\
& \mathcal{R}_{4}^{3}=\Gamma\left(S_{4}, Z_{6}, D_{4} ; Z_{2}, Z_{3}, Z_{2} ; 4,2,4\right), \\
& \mathcal{R}_{4}^{4}=\Gamma\left(\mathrm{Cu}_{24}, Z_{6}, D_{4} ; Z_{2}, Z_{3}, Z_{2} ; 4,2,4\right) .
\end{aligned}
$$

These groups are uniquely determined by the triangle of groups data. They are virtually torsion-free.

Proof. The group data follows immediately from the previous proposition and the previous lemmas.

The group $\mathcal{R}_{2}$ is the Coxeter group with diagram $\bullet{ }^{4} \bullet{ }^{4} \bullet$. The groups $\mathcal{R}_{3}^{1}$ and $\mathcal{R}_{4}^{1}$ are rotation subgroups of Coxeter groups with the diagrams

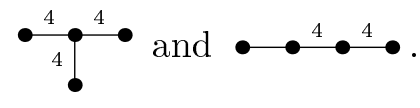

In fact, $\mathcal{R}_{4}^{1} \cong \mathrm{PGL}_{2}(Z[i])$.

The presentations for these groups are

$\mathcal{R}_{3}^{1}=\left\langle x, y, z \mid x^{2}, y^{2}, z^{3},(x y)^{2},(y z)^{4},(x z)^{4}\right\rangle$,

$\mathcal{R}_{3}^{2}=\left\langle x, y, z \mid x^{2}, y^{2}, z^{3},(x y)^{2},\left(y z y z^{-1}\right)^{2},\left(x z x z^{-1}\right)^{2}\right\rangle$,

$\mathcal{R}_{3}^{3}=\left\langle x, y, z \mid x^{2}, y^{2}, z^{3},(x y)^{2},(y z)^{4},\left(x z x z^{-1}\right)^{2}\right\rangle$,

$\mathcal{R}_{4}^{1}=\left\langle x, y, z \mid x^{2}, y^{3}, z^{2},(x z)^{4},(y z)^{2},(x y)^{4}\right\rangle$,

$\mathcal{R}_{4}^{2}=\left\langle x, y, z \mid x^{2}, y^{3}, z^{2},(x z)^{4},(y z)^{2},\left(x y x y^{-1}\right)^{2}\right\rangle$,

$\mathcal{R}_{4}^{3}=\left\langle x, y, z \mid x^{2}, y^{3}, z^{2},(x z)^{4}, z y z y^{-1},(x y)^{4}\right\rangle$,

$\mathcal{R}_{4}^{4}=\left\langle x, y, z \mid x^{2}, y^{3}, z^{2},(x z)^{4}, z y z y^{-1},\left(x y x y^{-1}\right)^{2}\right\rangle$.

If we change any edge identifications, say $z$ to $z^{-1}$ or $y$ to $y^{-1}$, we get an equivalent presentation. 
We collapse $z=1$ and fold $x=y$ to obtain homomorphisms

$$
\begin{aligned}
& \mathcal{R}_{3}^{1} \rightarrow D_{2} \times S_{4}, \\
& \mathcal{R}_{3}^{2} \rightarrow D_{2} \times \mathrm{Cu}_{24} .
\end{aligned}
$$

We first collapse $z=1$, then collapse $y=1$, then collapse $x=1$ to obtain a homomorphism

$$
\mathcal{R}_{3}^{3} \rightarrow D_{2} \times \mathrm{Cu}_{24} \times S_{4} .
$$

These three homomorphisms give injections on the vertex stabilizers for $\mathcal{R}_{3}^{i}$ for $i=1,2,3$.

Factoring $\mathcal{R}_{4}^{i}$ by the normal subgroup generated by $(x z y)^{k}$ for certain $k$ gives a finite group for which all vertex stabilizers inject: for $i=1,(x z y)^{4}$ gives a group of order 480; for $i=2,(x z y)^{2}$ gives a group of order 48; for $i=3,(x z y)^{4}$ gives a group of order 384 ; for $i=4,(x z y)^{5}$ gives a group of order 720 .

Proposition 6.6. A dihedral Platonic group with angles $\left[\frac{\pi}{6}, \frac{\pi}{2}, \frac{\pi}{3}\right]$ has order data given by one of

$$
\begin{aligned}
& \mathcal{B}_{2}=\Gamma(12,4,6 ; 2,2,2 ; 6,2,3), \\
& \mathcal{B}_{3}=\Gamma(12,6,12 ; 2,2,3 ; 6,2,3), \\
& \mathcal{B}_{4}=\Gamma(12,8,24 ; 2,2,4 ; 6,2,3), \\
& \mathcal{B}_{5}=\Gamma(12,10,60 ; 2,2,5 ; 6,2,3), \\
& \mathcal{B}_{8}=\Gamma(12,32,96 ; 2,2,8 ; 6,2,3), \\
& \mathcal{B}_{9}=\Gamma(12,54,108 ; 2,2,9 ; 6,2,3), \\
& \mathcal{B}_{10}=\Gamma(12,10 b, 10 c ; 2,2,10 ; 6,2,3),
\end{aligned}
$$

where

$(b, c) \in\{(7,42),(8,24),(9,18),(10,15),(12,12)\}$.

Proof. For the dihedral type, if the vertex group at angle $\frac{\pi}{2}$ has edges of order 2 , then $n \leq 3$ and so we get either $\mathcal{B}_{2}$ or there is a vertex $B \in \Gamma(3,2: 3)$ of order 12 by Lemma 4.7 , but this is impossible by Corollary 3.2. Therefore we may assume either that there is a vertex group $A \in \Gamma(2,2: 3)$ of order 6 or $A \in \Gamma(2,2: 6)$ of order 12 .

In the first case, $A \in \Gamma(2,2: 3)$ of order 6 , we have $B \in \Gamma(g, 2: 2)$ and $C \in \Gamma(g, 2: 6), 2<g<6$. Let $b=g b_{1}, c=g c_{1}$ so that $1-\frac{1}{b_{1}}-\frac{1}{c_{1}}=\frac{g}{6}$. If $g \in\{3,5\}$ then $b_{1}=2$ by Lemma 4.6 . Now by Corollary 3.2, these are impossible. If $g=4$, then $c_{1} \geq 26$ by Corollary 4.4 and hence this equation has no solutions.

Now consider the case of $A \in \Gamma(2,2: 6)$ of order 12. We have $B \in \Gamma(g, 2: 2)$ and $C \in \Gamma(g, 2: 3)$, with $2<g<12$. Let $b=g b_{1}, c=g c_{1}$ so that $1-\frac{1}{b_{1}}-\frac{1}{c_{1}}=\frac{g}{12}$. If $g \in\{3,5,7,11\}$, then $b_{1}=2$ by Lemma 4.6. If $g \in\{7,11\}$, then the equation above is not solvable. If $g=3$, then $c=12$; and if $g=5$, then $c=60$; this yields $\mathcal{B}_{3}^{1}, \mathcal{B}_{3}^{2}, \mathcal{B}_{5}^{1}, \mathcal{B}_{5}^{2}$. Now by Corollary 4.4, $C \in \Gamma(g, 2: 3)$ has order at least $g(g+1)$. If $g=4$, then $c_{1} \geq 5$ by Corollary 4.4 and hence $b_{1} \leq 2$ and therefore $b_{1}=2$ and $c_{1}=6$, which gives rise to the $\mathcal{B}_{4}$ family using Corollary 4.12 for the group of order 24 and Corollary 4.14 for the group of order 8 .

If $g=6$, then $\frac{1}{b_{1}}+\frac{1}{c_{1}}=\frac{1}{2}, b_{1} \geq 3, c_{1} \geq 7$, which has no solutions.

If $g=8$, then $\frac{1}{b_{1}}+\frac{1}{c_{1}}=\frac{1}{3}, b_{1} \geq 4, c_{1} \geq 9$, which has the solution $b_{1}=4, c_{1}=12$. This gives the family of groups $\mathcal{B}_{8}$.

If $g=9$, then $\frac{1}{b_{1}}+\frac{1}{c_{1}}=\frac{1}{4}, b_{1} \geq 5, c_{1} \geq 10$, which has the solutions $b_{1}=5, c_{1}=20$ and $b_{1}=6$, $c_{1}=12$, and this gives the family $\mathcal{B}_{9}$. However the vertex group $B$ has even order so we have only the case listed above.

If $g=10$, then $\frac{1}{b_{1}}+\frac{1}{c_{1}}=\frac{1}{6}, b_{1} \geq 7, c_{1} \geq 11$, which has solutions

$$
\left(b_{1}, c_{1}\right) \in\{(7,42),(8,24),(9,18),(10,15),(12,12)\} .
$$

This gives the family $\mathcal{B}_{10}$.

Theorem 6.7. A dihedral Platonic group with angles $\left[\frac{\pi}{6}, \frac{\pi}{2}, \frac{\pi}{3}\right]$ that is of geometric type is spherical and is one of the following:

$$
\begin{aligned}
& \mathcal{B}_{2}=\Gamma\left(D_{6}, D_{2}, D_{3} ; Z_{2}, Z_{2}, Z_{2} ; 6,2,3\right), \\
& \mathcal{B}_{3}^{1}=\Gamma\left(D_{6}, D_{3}, A_{4} ; Z_{2}, Z_{2}, Z_{3} ; 6,2,3\right), \\
& \mathcal{B}_{3}^{2}=\Gamma\left(D_{6}, Z_{6}, A_{4} ; Z_{2}, Z_{2}, Z_{3} ; 6,2,3\right), \\
& \mathcal{B}_{4}^{1}=\Gamma\left(D_{6}, D_{4}, S_{4} ; Z_{2}, Z_{2}, Z_{4} ; 6,2,3\right), \\
& \mathcal{B}_{4}^{2}=\Gamma\left(D_{6}, Z_{4} \times Z_{2}, S_{4} ; Z_{2}, Z_{2}, Z_{4} ; 6,2,3\right), \\
& \mathcal{B}_{2^{2}}^{1}=\Gamma\left(D_{6}, D_{2} \times Z_{2}, S_{4} ; Z_{2}, Z_{2}, D_{2} ; 6,2,3\right),
\end{aligned}
$$




$$
\begin{aligned}
& \mathcal{B}_{2^{2}}^{2}=\Gamma\left(D_{6}, D_{4}, S_{4} ; Z_{2}, Z_{2}, D_{2} ; 6,2,3\right), \\
& \mathcal{B}_{5}^{1}=\Gamma\left(D_{6}, D_{5}, A_{5} ; Z_{2}, Z_{2}, Z_{5} ; 6,2,3\right), \\
& \mathcal{B}_{5}^{2}=\Gamma\left(D_{6}, Z_{10}, A_{5} ; Z_{2}, Z_{2}, Z_{5} ; 6,2,3\right) .
\end{aligned}
$$

These triangles of groups are uniquely determined by the group data. All of these triangles of groups are virtually torsion-free.

Proof. The geometric type restriction (4-1) (see page 197) implies that the families $\mathcal{B}_{8}, \mathcal{B}_{9}, \mathcal{B}_{10}$ do not occur. The data for each of the families follows easily from the previous proposition and proof. It is easy to show that the groups are uniquely determined by the data.

The group $\mathcal{B}_{2}$ is the Coxeter group with diagram $\bullet{ }^{6} \bullet$. The groups $\mathcal{B}_{3}^{1}, \mathcal{B}_{4}^{1}, \mathcal{B}_{5}^{1}$ are rotation subgroups of the Coxeter groups with respective diagrams $\bullet \bullet \bullet^{6} \bullet, \bullet^{4} \bullet \bullet^{6} \bullet, \bullet^{5} \bullet \bullet^{6} \bullet$. In fact, we have $\mathcal{B}_{3}^{1} \cong \operatorname{PGL}_{2}\left(Z\left[\frac{1}{2}(1+\sqrt{-3})\right]\right)$. The group $\mathcal{B}_{2^{2}}^{1}$ is the Coxeter group with diagram

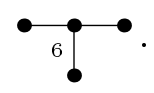

In the groups of the families $\mathcal{B}_{4}, \mathcal{B}_{2^{2}}$ we use the presentation

$$
S_{4}=\left\langle x, z, w \mid x^{2}, w^{2}, z^{2},(w z)^{2},(x w)^{3},(x z)^{3}\right\rangle .
$$

The presentations for these groups are

$$
\begin{aligned}
& \mathcal{B}_{3}^{1}=\left\langle x, y, z \mid x^{2}, y^{2}, z^{3},(x y)^{6},(y z)^{2},(x z)^{3}\right\rangle, \\
& \mathcal{B}_{3}^{2}=\left\langle x, y, z \mid x^{2}, y^{2}, z^{3},(x y)^{6}, y z y z^{-1},(x z)^{3}\right\rangle, \\
& \mathcal{B}_{4}^{1}=\left\langle x, y, z \mid x^{2}, y^{2}, z^{4},(x y)^{6},(y z)^{3},(x z)^{2}\right\rangle, \\
& \mathcal{B}_{4}^{2}=\left\langle x, y, z \mid x^{2}, y^{2}, z^{4},(x y)^{6},(y z)^{3}, x z x z^{-1}\right\rangle, \\
& \mathcal{B}_{5}^{1}=\left\langle x, y, z \mid x^{2}, y^{2}, z^{5},(x y)^{6},(y z)^{2},(x z)^{3}\right\rangle, \\
& \mathcal{B}_{5}^{2}=\left\langle x, y, z \mid x^{2}, y^{2}, z^{5},(x y)^{6}, y z y z^{-1},(x z)^{3}\right\rangle, \\
& \mathcal{B}_{2^{2}}^{1}=\langle x, y, z, w| x^{2}, y^{2}, z^{2}, w^{2},(z w)^{2}, \\
& \left.\quad(x y)^{6},(x z)^{3},(x w)^{3},(y z)^{2},(y w)^{2}\right\rangle, \\
& \mathcal{B}_{2^{2}}^{2}=\langle x, y, z, w| x^{2}, y^{2}, z^{2}, w^{2},(z w)^{2}, \\
& \left.\quad(x y)^{6},(x z)^{3},(x w)^{3},(y z)^{4}, w,(y z)^{2}\right\rangle .
\end{aligned}
$$

These groups are determined by the group data since, for example, changing $z$ to $z^{-1}$ leads to an equivalent presentation for $\mathcal{B}_{i}$, for $i=3,4,5$. In the case of $\mathcal{B}_{2^{2}}^{2}$, other than the relation $(y z)^{4}=1$ in $D_{4}$ we could have another element of order 2 , $w=(y z)^{2}, w=y z y$ or $w=z y z$ but in the latter two cases the angle is not correct.

Factoring $\mathcal{B}$ by the normal subgroup generated by $(x z y)^{k}$ for certain $k$ gives a finite group for which all vertex stabilizers inject: for $\mathcal{B}_{3}^{1},(x z y)^{4}$ gives a group of order 120; for $\mathcal{B}_{3}^{2},(x z y)^{4}$ gives a group of order 1008 ; for $\mathcal{B}_{4}^{1},(x z y)^{2}$ gives a group of order 48 ; for $\mathcal{B}_{4}^{2},(x z y)^{4}$ gives a group of order 384 ; for $\mathcal{B}_{5}^{1},(x z y)^{4}$ gives a group of order 6840 ; for $\mathcal{B}_{5}^{2},(x z y)^{4}$ gives a group of order 14400 . In the case of $\mathcal{B}_{5}^{1}$, we can also obtain a permutation representation $\mathcal{B}_{5}^{1} \rightarrow S_{10}, x \rightarrow(24)(35)(69)(710)$, $y \rightarrow(12)(36)(47)(59)(810), z \rightarrow(135107)(24896)$, which is injective on the vertex stabilizers. The image is of order 120 so this gives a minimal index torsion-free subgoup, since $A_{5}$ has no elements of order 6 . This is related to a question raised in [Milnor 1994].

Factoring $\mathcal{B}_{2^{2}}^{1}$ by the normal subgroup generated by $(y w x z)^{4}$ gives a group of order 192 for which all vertex stabilizers inject. Factoring $\mathcal{B}_{2^{2}}^{2}$ by the normal subgroup generated by $(x y z)^{6}$ gives a group of order 34560 for which all vertex stabilizers inject. In this last case, we can also obtain a surjective permutation representation $\mathcal{B}_{2^{2}}^{2} \rightarrow S_{6}, x \rightarrow(23)(56)$, $y \rightarrow(24), z \rightarrow(12)(45)$, which is injective on the vertex stabilizers.

\section{Octahedral}

The finite groups in the family $\Gamma(3,2: 6)$ have been considered by Burnside and Coxeter. There are three subgroups of index 2 in the reflection group of a triangle with angles $\frac{\pi}{2}, \frac{\pi}{3}, \frac{\pi}{6}$; the rotation subgroup

$$
\left\langle x, y \mid x^{2}, y^{3},(x y)^{6}\right\rangle
$$

the reflections in the doubled triangle, with angles $\frac{\pi}{3}, \frac{\pi}{3}, \frac{\pi}{3}$,

$$
\left\langle x, y, z \mid x^{2}, y^{2}, z^{2},(x y)^{3},(y z)^{3},(z x)^{3}\right\rangle ;
$$


and the group generated by a rotation of order three at the vertex of an isosceles triangle with angle $\frac{2 \pi}{3}$ and the reflection in the opposite side,

$$
\left\langle x, y \mid x^{2}, y^{3},\left(x y x y^{-1}\right)^{3}\right\rangle .
$$

The finite quotients are related to representations of integers by the norm form

$$
a^{2}+a b+b^{2}=(a+b \omega)\left(a+b \omega^{-1}\right)
$$

in the quadratic field $Q(\omega), \omega^{2}=\omega-1$. Note that two solutions to $a^{2}+a b+b^{2}=m$ in integers have ratio in $Q(\omega)$ of norm 1 and hence the ratio is a power of $\omega$. Multiplication by $\omega$ takes the solution $a+b \omega$ to $-b+(a+b) \omega$.

Lemma 6.8. The groups in $\Gamma(3,2: 6)$ are of order 42 , 48 or obtained from amalgam quotients of certain 2-dimensional crystallographic groups having order $6 m$, where $m=a^{2}+a b+b^{2}$, for integers $a, b$.

Proof. Consider the amalgam quotients of $Z_{3} * Z_{2}$ having $y, x$ as the generators of the factors. The possible relations of length 6 after changing $y$ to $y^{-1}$ if necessary are $(x y)^{6},(x y)^{5} x y^{-1},(x y)^{4}\left(x y^{-1}\right)^{2}$, $\left((x y)^{2} x y^{-1}\right)^{2},(x y)^{3}\left(x y^{-1}\right)^{3},(x y)^{3}\left(x y^{-1}\right)(x y)\left(x y^{-1}\right)$, $(x y)^{2}\left(x y^{-1}\right)(x y)\left(x y^{-1}\right)^{2},\left(x y x y^{-1}\right)^{3}$. The groups obtained from these relations have orders $\infty, 2$, $6,48,48,2,42, \infty$. Thus we get amalgams of the virtually free abelian (rank 2) groups

$$
\mathrm{Cu}_{(2,3,6)}^{1}=\left\langle x, y \mid x^{2}, y^{3},(x y)^{6}\right\rangle
$$

and

$$
\mathrm{Cu}_{(2,3,6)}^{2}=\left\langle x, y \mid x^{2}, y^{3},\left(x y x y^{-1}\right)^{3}\right\rangle .
$$

These amalgams have been classified by Burnside [1911, p. 419] and Sinkov [1936, Theorem 7]. According to Burnside, the finite amalgams of the 2,3,6 triangle group $\mathrm{Cu}_{(2,3,6)}^{1}$ are the groups

$\mathrm{Cu}_{\langle a, b\rangle}=\left\langle x, y \mid x^{2}, y^{3},(x y)^{6},\left(y x y^{-1} x\right)^{a}\left(y^{-1} x y x\right)^{b}\right\rangle$

of order $6 m=6\left(a^{2}+a b+b^{2}\right)$, and different solutions to $m=\left(a^{2}+a b+b^{2}\right)$ give the same group.
According to Sinkov, the finite amalgams of $\mathrm{Cu}_{(2,3,6)}^{2}$ are

$$
\mathrm{Cu}_{(b, c)}=\left\langle x, y \mid x^{2}, y^{3},\left(x y x y^{-1}\right)^{3},(y x)^{2 b}\left(y^{-1} x\right)^{2 c}\right\rangle
$$

of order $6\left(b^{2}+b c+c^{2}\right)$. We can see that we get the same group for a different solution to the norm equation as follows. First, it follows from the commutator equation that $(x y)^{2}$ commutes with $(y x)^{2}$ and hence also $(x y)^{-2}(y x y)^{2}=(y x)^{2}$. Repeatedly applying this relation yields

$$
(x y)^{-2 c}(y x y)^{2(b+c)}=(y x)^{2 c}(y x y)^{2 b} .
$$

It follows by a conjugation by $y^{-1}$ that

$$
(y x)^{2 b}\left(y^{-1} x\right)^{2 c}=1 \Longleftrightarrow(y x)^{2 c}(y x y)^{2 b}=1
$$

or equivalently, by a conjugation, that $(y x)^{2 c}=$ $\left(y^{-1} x\right)^{2(b+c)}$.

The others obtained are

$$
\mathrm{Cu}_{42}=\left\langle x, y \mid x^{2}, y^{3},(x y)^{2}\left(x y^{-1}\right)(x y)\left(x y^{-1}\right)^{2}\right\rangle,
$$

and two groups of order 48, distinguished by the order of $x y$, either 8 or 12 :

$$
\begin{aligned}
& \mathrm{Cu}_{48}^{a}=\left\langle x, y \mid x^{2}, y^{3},\left((x y)^{2} x y^{-1}\right)^{2}\right\rangle, \\
& \mathrm{Cu}_{48}^{b}=\left\langle x, y \mid x^{2}, y^{3},(x y)^{3}\left(x y^{-1}\right)^{3}\right\rangle .
\end{aligned}
$$

Corollary 6.9. The groups of order $48,54,72$ and 96 in $\Gamma\left(Z_{3}, Z_{2}: 6\right)$ are $\mathrm{Cu}_{48}^{a}, \mathrm{Cu}_{48}^{b}, \mathrm{Cu}_{54}, \mathrm{Cu}_{72}^{a}, \mathrm{Cu}_{72}^{b}$, $\mathrm{Cu}_{96}^{a}, \mathrm{Cu}_{96}^{b}$ with the presentations given below. In the family, $\Gamma\left(Z_{3}, Z_{2}: 6\right)$, there are no groups of order divisible by 5 but not 25.

Proof. According to Lemma 6.8, the groups of order 48 in $\Gamma(3,2: 6)$ are then $\mathrm{Cu}_{48}^{a}, \mathrm{Cu}_{48}^{b}$. The groups of order 54,72, 96 are either $\mathrm{Cu}_{(c, d)}, c^{2}+d^{2}+c d=$ $9,12,16$ or $\mathrm{Cu}_{\langle a, b\rangle}, a^{2}+b^{2}+a b=9,12,16$. According to the arguments above the groups $\mathrm{Cu}_{(c, d)}$ of order $6 m$ are isomorphic for any solution to $c^{2}+c d+d^{2}=m$; similar arguments apply for $\mathrm{Cu}_{\langle a, b\rangle}$. It follows that the group of order 54 is uniquely determined,

$$
\mathrm{Cu}_{54}=\left\langle x, y \mid x^{2}, y^{3},(x y)^{6},\left(y x y^{-1} x\right)^{3}\right\rangle .
$$


There are two groups of order 72, distinguished by their derived series:

$$
\begin{aligned}
& \mathrm{Cu}_{72}^{a}=\left\langle x, y \mid x^{2}, y^{3},(x y)^{6},\left(y x y^{-1} x\right)^{2}\left(y^{-1} x y x\right)^{2}\right\rangle, \\
& \mathrm{Cu}_{72}^{b}=\left\langle x, y \mid x^{2}, y^{3},\left(x y x y^{-1}\right)^{3},(y x)^{4}\left(y^{-1} x\right)^{4}\right\rangle .
\end{aligned}
$$

There are two groups of order 96, distinguished by their derived series:

$$
\begin{aligned}
& \mathrm{Cu}_{96}^{a}=\left\langle x, y \mid x^{2}, y^{3},(x y)^{6},\left(x y^{-1} x y\right)^{4}\right\rangle, \\
& \mathrm{Cu}_{96}^{b}=\left\langle x, y \mid x^{2}, y^{3},\left(x y x y^{-1}\right)^{3},(x y)^{8}\right\rangle .
\end{aligned}
$$

Note that, since $a^{2}+a b+b^{2} \bmod 5$ has no solutions except $a=b=0 \bmod 5$, none of these orders are divisible by 5 but not 25 . Further results for powers of 2 or odd powers of 5 can also be easily deduced.

Proposition 6.10. The octahedral flat Platonic groups having an angle of $\frac{\pi}{2}$ have triangle and vertex data as specified below:

$$
\begin{aligned}
& \mathcal{O}_{1}^{(n, l)}=\Gamma\left(S_{4}, 4 n, 12 l: 3,2,4: 4,2,4\right), \\
& \mathcal{O}_{2}^{(n, l)}=\Gamma\left(\mathrm{Cu}_{24}, 4 n, 12 l: 3,2,4: 4,2,4\right),
\end{aligned}
$$

for $(n, l) \in\{(7,14),(8,8),(9,6),(10,5),(12,4)$, $(18,3)\}$

$$
\begin{aligned}
\mathcal{O}_{3}^{(m, k)} & =\Gamma\left(\mathrm{Cu}_{18}, 4 m, 12 k: 3,2,4: 4,2,4\right), \\
\text { for }(m, k) \in & \{(10,30),(12,12)\} ; \\
\mathcal{O}_{4}^{(n, l)} & =\Gamma\left(S_{4}, 4 n, 12 l: 3,2,4: 4,4,2\right), \\
\mathcal{O}_{5}^{(n, l)} & =\Gamma\left(\mathrm{Cu}_{24}, 4 n, 12 l: 3,2,4: 4,4,2\right),
\end{aligned}
$$

for $(n, l) \in\{(7,14),(8,8),(9,6),(10,5),(12,4)$, $(18,3)\}$;

$$
\begin{gathered}
\mathcal{O}_{6}^{(m, k)}=\Gamma\left(\mathrm{Cu}_{18}, 4 m, 12 k: 3,2,4: 2,4,4\right), \\
\text { for }(m, k) \in\{(10,30),(12,12),(18,6)\} ; \\
\mathcal{O}_{7}^{(r, s)}=\Gamma\left(S_{4}, 6 r, 4 s: Z_{4}, Z_{3}, Z_{2}: 2,6,3\right), \\
\mathcal{O}_{8}^{(r, s)}=\Gamma\left(S_{4}, 6 r, 4 s: D_{2}, Z_{3}, Z_{2}: 2,6,3\right), \\
\mathcal{O}_{9}^{(r, s)}=\Gamma\left(\mathrm{SL}_{2}\left(Z_{3}\right), 6 r, 4 s: Z_{4}, Z_{3}, Z_{2}: 2,6,3\right), \\
\mathcal{O}_{10}^{(r, s)}=\Gamma\left(\mathrm{Cu}_{24}, 6 r, 4 s: D_{2}, Z_{3}, Z_{2}: 2,6,3\right),
\end{gathered}
$$

$$
\begin{aligned}
\text { for }(r, s) \in\{(8,12),(12,9)\} ; \text { or } \\
\mathcal{O}_{11}=\Gamma(48,48,16: 4,3,2: 3,6,2), \\
\mathcal{O}_{12}=\Gamma(36,54,16: 4,3,2: 3,6,2), \\
\mathcal{O}_{13}=\Gamma(96,72,16: 4,3,2: 3,6,2), \\
\mathcal{O}_{14}=\Gamma(432,96,16: 4,3,2: 3,6,2), \\
\mathcal{O}_{15}=\Gamma(144,72,24: 4,3,2: 3,6,2) .
\end{aligned}
$$

Proof. In the octahedral case the vertex of angle $\frac{\pi}{2}$ has edges of orders 2 and 4 or 3 and 4 , since otherwise by Lemma 4.6 the vertex order would be of order 6 contradicting Corollary 3.2. There are two cases for the arrangement of angles depending on which is the angle $\frac{\pi}{2}$ according to the previous remarks.

In case the triangle is isosceles, there is a vertex in $\Gamma(3,2: 4)$ of order 18 or 24 . This gives rise to equations $\frac{1}{12}=\frac{1}{18}+\frac{1}{4 m}+\frac{1}{12 k}$ or $\frac{1}{12}=\frac{1}{24}+\frac{1}{4 n}+\frac{1}{12 l}$. Simplifying this gives the equations

$$
1=\frac{6}{n}+\frac{2}{l}, \quad 1=\frac{9}{m}+\frac{3}{k}
$$

An easy analysis yields the solutions to the first equation:

$(n, l) \in\{(7,14),(8,8),(9,6),(10,5),(12,4),(18,3)\}$.

The solutions to the second equation are $(m, k) \in$ $\{(10,30),(12,12),(18,6)\}$. There are two cases for the edge groups of order 4 . Notice, however, that $l, k \geq 7$ in case the vertex group is in $\Gamma(4,3: 4)$ by Corollary 4.4 .

These calculations give the families $\mathcal{O}_{i}$, for $i=$ $1, \ldots, 6$.

If the triangle is not isoceles we obtain the more general equation, $\frac{1}{12}=\frac{1}{6 r}+\frac{1}{4 s}+\frac{1}{12 t}$, rewritten as

$$
1=\frac{2}{r}+\frac{3}{s}+\frac{1}{t} \text {. }
$$

Thus $t \geq 2$. Again, there are two cases for the vertex angle $\frac{\pi}{2}$. Also, since a group in $\Gamma(3,2: 3)$ has order 12 and a group in $\Gamma(3,2: 2)$ has order 6 , it must be the case that the angle between edges of orders 2 and 3 is $\frac{\pi}{6}$. In this case the vertex group in $\Gamma(3,2: 6)$ has order $6 r, r \geq 7$. 
Now a vertex group in $\Gamma(4,2: 3)$ has order $4 s$, $s \geq 8$, by Corollary 4.4. A vertex group in $\Gamma(4,3: 2)$ has order $12 t, t \geq 2$. Since $\frac{2}{7}+\frac{3}{8}+\frac{1}{t}<1$, for $t \geq 3$, it follows that the only solutions are for $t=2$ and these are easily determined as

$$
(r, s) \in\{(7,14),(8,12),(10,10),(12,9),(16,8)\} .
$$

But now, by Lemma 4.11, the groups of $\Gamma(4,2: 3)$ of order greater than 20 have order divisible by 12 and thus $s$ is a multiple of 3 . Thus the solutions are $(r, s) \in\{(8,12),(12,9)\}$. This gives the family of groups $\mathcal{O}_{i}, i=7, \ldots, 10$. The groups of order $48, r=8$, and of order $72, r=12$, in $\Gamma(3,2: 6)$ are described above. The groups of order $48, s=$ 12 , and of order $36, s=9$, in $\Gamma(4,2: 3)$ can be determined by Lemma 4.11 and Proposition 4.10.

Next, by the remarks above, there is no vertex group $\Gamma(3,2: 3)$, so we must have a vertex in $\Gamma(3,2: 6)$ of order $6 r, r \geq 7$, by Corollary 4.4. Also, there is a vertex group $\Gamma(4,3: 3)$ of order $12 t, t \geq 3$, by Corollary 4.4 and a vertex group in $\Gamma(4,2: 2)$ of order $4 s, s \geq 4$ by Corollary 3.2.

Since $\frac{2}{7}+\frac{3}{8}+\frac{1}{3}<1$, it follows that the solutions occur for $r \geq 7,4 \leq s \leq 7, t \geq 3$. Analyzing by cases we find that for $s=7, r \geq 7, \frac{2}{r}+\frac{1}{t}=\frac{4}{7}$, then $t \leq 3$ and hence no solutions; for $s=6, r \geq 7$, $\frac{2}{r}+\frac{1}{t}=\frac{1}{2}$, then $t \leq 4$ and hence solutions $(r, t) \in$ $\{(12,3),(8,4)\}$; for $s=5, r \geq 7, \frac{2}{r}+\frac{1}{t}=\frac{2}{5}$, then $t \leq 9$ and hence solutions $(r, t) \in\{(30,3),(10,5)\}$; for $s=4, r \geq 9, \frac{2}{r}+\frac{1}{t}=\frac{1}{4}$, then $t \leq 36$ and hence solutions $(r, t) \in\{(9,36),(10,20),(12,12)$, $(16,8),(24,6),(40,5)\}$. By the remarks above on the representations of integers by the form $a^{2}+$ $a b+b^{2}$ we see that $r=10,24,30,40$ are impossible. This gives the solutions $(r, s, t) \in\{(8,6,4),(9,4,36)$, $(12,4,12),(12,6,3),(16,4,8)\}$. The groups of order 16 and 24 in $\Gamma(4,2: 2)$ are then easily determined from Lemma 4.11 .

Proposition 6.11. (i) The finite amalgam of $D_{3} \underset{Z_{2}}{*} D_{2}$ in $\Gamma(4,2: 3)$ of order 48 is the Coxeter reflection group

$$
B_{48}=\left\langle x, y, z \mid x^{2}, y^{2}, z^{2},(x y)^{3},(y z)^{2},(x z)^{4}\right\rangle .
$$

(ii) There are no amalgams of order 48 of $D_{3} \underset{Z_{2}}{*} Z_{4}$ in $\Gamma(4,2: 3)$.

Proof. The abelianization of these two amalgams are (i) $D_{2}$ or (ii) $Z_{4}$ respectively, and hence the abelianization of any finite quotient is a 2-group of order at most 4. By Proposition 4.10 the image of the cyclic subgroup of order 3 is not normal, hence there are either 4 or 16 3-Sylow subgroups. If there are 16, then this leads to 32 elements of order 3 and hence the 2-Sylow subgroup is normal, contradicting the abelianization result above. Therefore we obtain a homomorphism of the amalgam $G, G \rightarrow S_{4}$. Now the normalizer of a 3-Sylow subgroup has order 12 and any two can meet only on a subgroup of order 2 or 4 . Hence the kernel $K$ of this homomorphism is either of order 2 or 4 . If $K$ is of order 4, then the image of the homomorphism is $A_{4}$. In this case lifting the 2-Sylow subgroup of $A_{4}$ back to $G$ gives a normal 2-Sylow subgroup, contradicting the abelianization result. Thus $G$ is an extension of $S_{4}$ by a normal subroup of order 2 (hence central). Now in the first case i) we have by Proposition 4.10 that there are no amalgam quotients of order 24 . Now in the second case the image of $x z$ in $S_{4}$ has order 2,3 , or 4 . However the group

$$
\left\langle x, y, z \mid x^{2}, y^{2}, z^{2},(x y)^{3},(y z)^{2},(x z)^{2}\right\rangle
$$

has order 12 and the group

$$
\left\langle x, y, z \mid x^{2}, y^{2}, z^{2},(x y)^{3},(y z)^{2},(x z)^{3}\right\rangle
$$

has order 24. Thus, since

$$
\left\langle x, y, z \mid x^{2}, y^{2}, z^{2},(x y)^{3},(y z)^{2},(x z)^{4}\right\rangle
$$

has order 48 , this is the uniquely determined amalgam.

Proposition 6.12. (i) Any finite amalgam of $D_{3} \underset{Z_{2}}{*} D_{2}$ in $\Gamma(4,2: 3)$ of order 36 is

$F_{36}^{a}=\left\langle x, y, z \mid x^{2}, y^{2}, z^{2},(x y)^{3},(y z)^{2},(x y z)^{2}(y x z)^{2}\right\rangle$. 
(ii) Any finite amalgam of $D_{3} \underset{Z_{2}}{*} Z_{4}$ in $\Gamma(4,2: 3)$ of order 36 is

$$
F_{36}^{b}=\left\langle x, y \mid x^{2}, y^{4},\left(x y^{2}\right)^{3},(x y)^{4}\right\rangle .
$$

Proof. Since the abelianizations are 2-groups of order less than 4, the 2-Sylow subgroups are not normal. If there are four Sylow 3-subgroups, then they are abelian and equal to their normalizers so by the normal $p$-complement theorem there is a normal 2complement. Thus the Sylow 3-subgroup is normal and the group is a split extension. If there is an element of order 9 then there is a unique element of order 3. The group of order 4 acts via inversion, so that the element of order 3 is fixed, thus giving a normal subgroup of order 3 contradicting Proposition 4.10. Thus the group is a split extension of $Z_{3}^{2}$ by $D_{2}$ or $Z_{4}$ depending on case (i) or (ii).

In the first case, if the $D_{2}$ acts trivially on one of the $Z_{3}$ factors we get a $Z_{6}$ homomorphic image contradiciting the abelianization. Thus the group is isomorphic to $S_{3} \times S_{3}$. Since $D_{2}=\langle y, z\rangle, x y$ is of order 3 and so is $z x y z$, these two elements commute: $x y z x y z=z x y z x y$. Addition of this relation suffices to give the group of order 36 ,

$$
\left\langle x, y, z \mid x^{2}, y^{2}, z^{2},(x y)^{3},(y z)^{2},(x y z)^{2}(y x z)^{2}\right\rangle .
$$

In the second case to avoid a $Z_{3}$ factor, the $Z_{4}$ acts by interchanging the $Z_{3}$ factors. The element $y$ conjugates $x y^{2}$ of order 3 to $y x y$, and these commute to give the group

$$
\left\langle x, y \mid x^{2}, y^{4},\left(x y^{2}\right)^{3},(x y)^{4}\right\rangle .
$$

Proposition 6.13. (i) The coset graphs $G(48: 3,2: 6)$, $G(72: 3,2: 6)$ of Corollary 6.9 are toroidal.

(ii) The coset graph $G(48: 4,2: 3)$ of Proposition 6.11 is planar.

(iii) The coset graphs $G(36: 4,2: 3)$ of Proposition 6.12 are imbeddable in $P^{2}$.

Proof. The graphs of the two groups of order 48 are isomorphic. Realizing the graph as having 24 (barycentrically marked) edges and 16 vertices of degree 3 , we imbed it in the torus as 8 hexagons. See Figure 5.
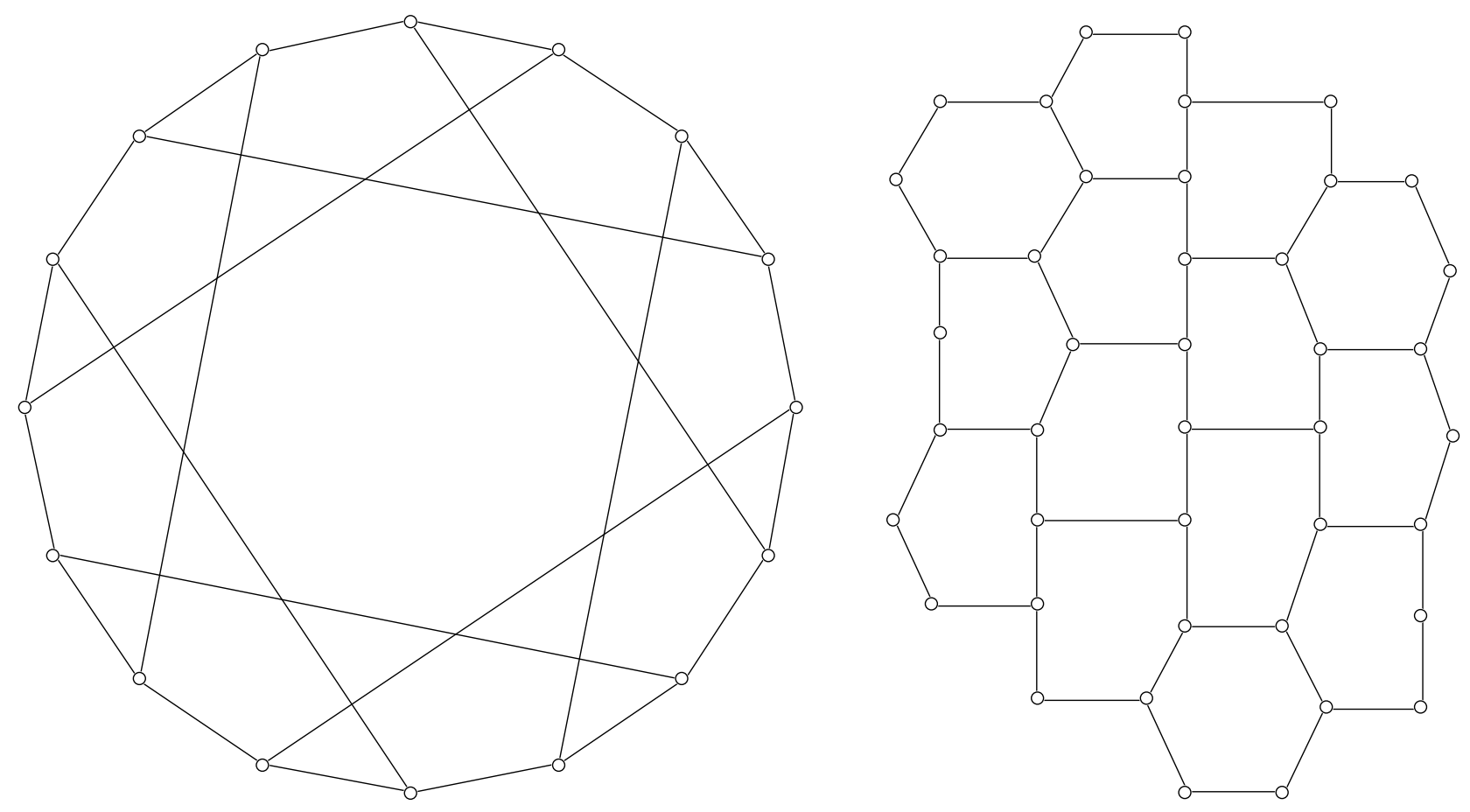

FIGURE 5. Left: $G(48: 3,2: 6)$. Right: The same graph on the torus (hexagonal identifications). 

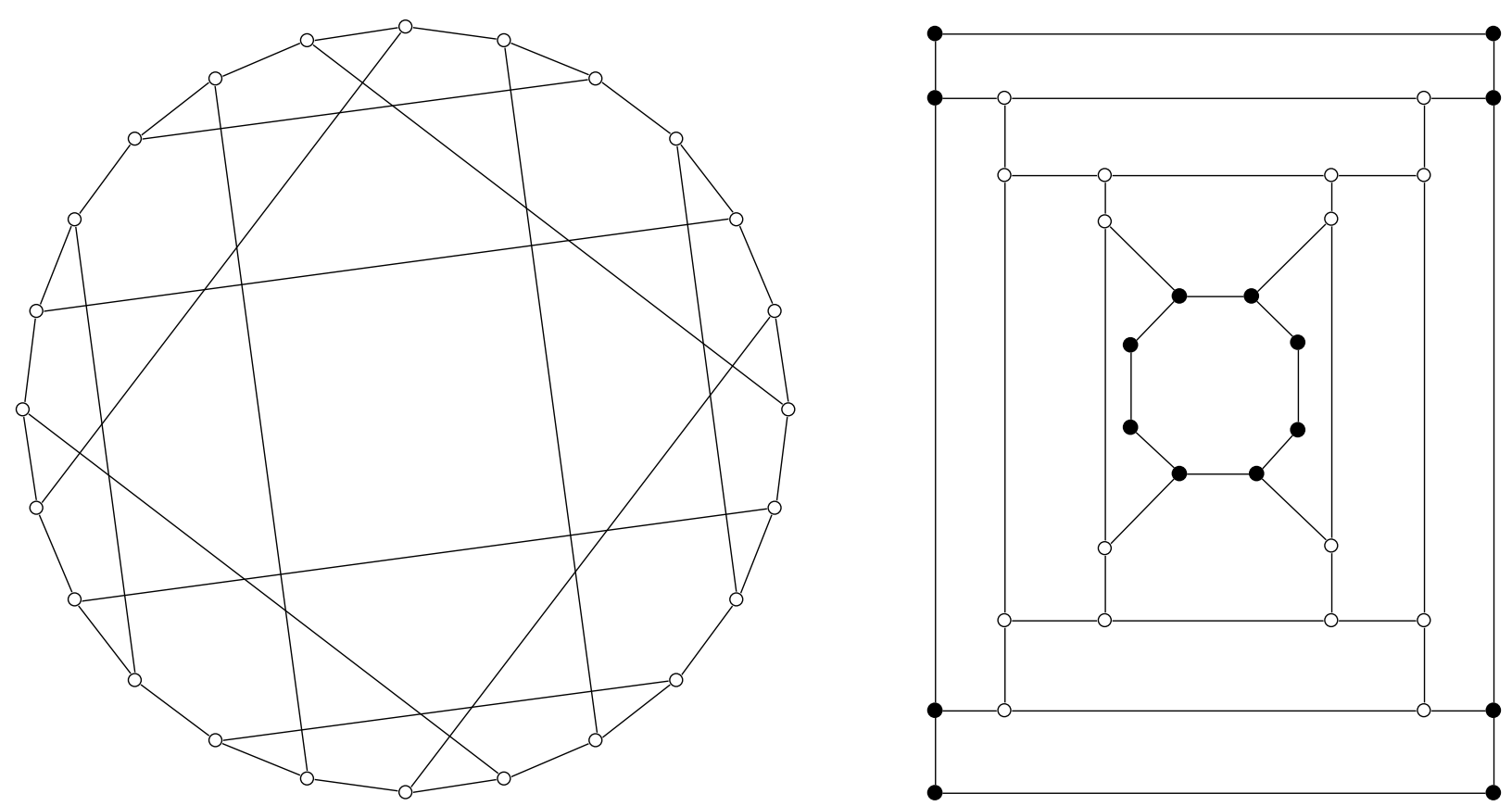

FIGURE 6. Left: $G(72: 3,2: 6)$. Right: The same graph on the torus (octagonal identifications).

The graphs of the two groups of order 72 are isomorphic. Realizing the graph as having 36 (barycentrically marked) edges and 24 vertices of degree 3 , we imbed it in the torus as 12 hexagons. See Figure 6.

The graph of Proposition 6.11 is obtained from two copies of an 8-gon attached to a 16-gon along alternate and every fourth vertex, respectively. The two copies are attached along the 16-gons at every fourth vertex symmetrically centered between the other fourths. See Figure 7.

The graphs of Proposition 6.12 of groups of order 36 are isomorphic and easily imbeddable in $P^{2}$, but not in the plane. See Figure 8.

Theorem 6.14. The octahedral flat Platonic groups having an angle of $\frac{\pi}{2}$ that are of geometric type are toroidal and have triangle and vertex data

$$
\begin{aligned}
& \mathcal{O} g_{1}=\Gamma\left(\mathrm{Cu}_{24}, B, B_{48}: D_{2}, Z_{3}, Z_{2}: 2,6,3\right), \\
& \mathcal{O} g_{2}=\Gamma\left(\mathrm{Cu}_{24}, B, F_{36}^{a}: D_{2}, Z_{3}, Z_{2}: 2,6,3\right), \\
& \mathcal{O} g_{3}=\Gamma\left(S_{4}, B, F_{36}^{b}: Z_{4}, Z_{3}, Z_{2}: 2,6,3\right),
\end{aligned}
$$

for $B \in\left\{\mathrm{Cu}_{48}^{a}, \mathrm{Cu}_{48}^{b}, \mathrm{Cu}_{72}^{a}, \mathrm{Cu}_{72}^{b}\right\}$.

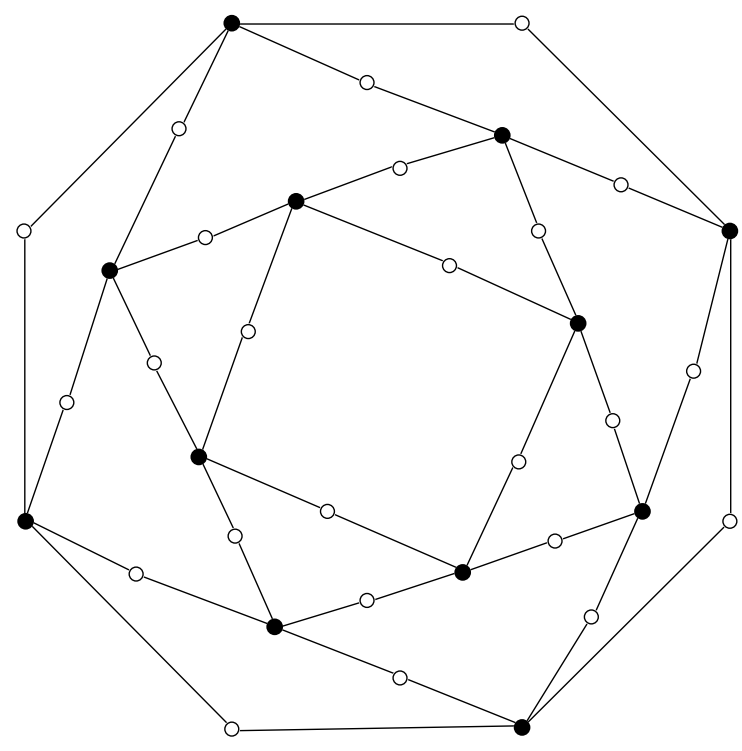

FIGURE 7. $G(48: 4,2: 3)$.

The presentations of these groups are easily determined, and are shown at the top of the next page.

All these groups are virtually torsion-free. They each have a subgroup of index less than or equal to 18 that yields a normal core which is torsion-free. 


$$
\begin{aligned}
& \mathcal{O} g_{1}^{p}\left\langle x, y, z, w \mid x^{2}, y^{3}, z^{2},(w z)^{2}, w^{2},(x z)^{3},(x w)^{4}, y z y^{-1} w,\left((x y)^{2} x y^{-1}\right)^{2}\right\rangle \\
& \mathcal{O} g_{1}^{q}\left\langle x, y, z, w \mid x^{2}, y^{3}, z^{2},(w z)^{2}, w^{2},(x z)^{3},(x w)^{4}, y z y^{-1} w,(x y)^{3}\left(x y^{-1}\right)^{3}\right\rangle \\
& \mathcal{O} g_{1}^{r}\left\langle x, y, z, w \mid x^{2}, y^{3}, z^{2},(w z)^{2}, w^{2},(x z)^{3},(x w)^{4}, y z y^{-1} w,(x y)^{6},\left(y x y^{-1} x\right)^{2}\left(y^{-1} x y x\right)^{2}\right\rangle \\
& \mathcal{O} g_{1}^{s}\left\langle x, y, z, w \mid x^{2}, y^{3}, z^{2},(w z)^{2}, w^{2},(x z)^{3},(x w)^{4}, y z y^{-1} w,\left(x y x y^{-1}\right)^{3},(y x)^{4}\left(y^{-1} x\right)^{4}\right\rangle \\
& \mathcal{O} g_{2}^{p}\left\langle x, y, z, w \mid x^{2}, y^{3}, z^{2},(w z)^{2}, w^{2},(x z)^{3},(x z w)^{2}(z x w)^{2}, y z y^{-1} w,\left((x y)^{2} x y^{-1}\right)^{2}\right\rangle \\
& \mathcal{O} g_{2}^{q}\left\langle x, y, z, w \mid x^{2}, y^{3}, z^{2},(w z)^{2}, w^{2},(x z)^{3},(x z w)^{2}(z x w)^{2}, y z y^{-1} w,(x y)^{3}\left(x y^{-1}\right)^{3}\right\rangle \\
& \mathcal{O} g_{2}^{r}\left\langle x, y, z, w \mid x^{2}, y^{3}, z^{2},(w z)^{2}, w^{2},(x z)^{3},(x z w)^{2}(z x w)^{2}, y z y^{-1} w,(x y)^{6},\left(y x y^{-1} x\right)^{2}\left(y^{-1} x y x\right)^{2}\right\rangle \\
& \mathcal{O} g_{2}^{s}\left\langle x, y, z, w \mid x^{2}, y^{3}, z^{2},(w z)^{2}, w^{2},(x z)^{3},(x z w)^{2}(z x w)^{2}, y z y^{-1} w,\left(x y x y^{-1}\right)^{3},(y x)^{4}\left(y^{-1} x\right)^{4}\right\rangle \\
& \mathcal{O} g_{3}^{p}\left\langle x, y, z \mid x^{2}, y^{3}, z^{4},(y z)^{2},\left(x z^{2}\right)^{3},(x z)^{4},\left((x y)^{2} x y^{-1}\right)^{2}\right\rangle \\
& \mathcal{O} g_{3}^{q}\left\langle x, y, z \mid x^{2}, y^{3}, z^{4},(y z)^{2},\left(x z^{2}\right)^{3},(x z)^{4},(x y)^{3}\left(x y^{-1}\right)^{3}\right\rangle \\
& \mathcal{O} g_{3}^{r}\left\langle x, y, z \mid x^{2}, y^{3}, z^{4},(y z)^{2},\left(x z^{2}\right)^{3},(x z)^{4},(x y)^{6},\left(y x y^{-1} x\right)^{2}\left(y^{-1} x y x\right)^{2}\right\rangle \\
& \mathcal{O} g_{3}^{s}\left\langle x, y, z \mid x^{2}, y^{3}, z^{4},(y z)^{2},\left(x z^{2}\right)^{3},(x z)^{4},\left(x y x y^{-1}\right)^{3},(y x)^{4}\left(y^{-1} x\right)^{4}\right\rangle
\end{aligned}
$$

Presentations for the groups in Theorem 6.14.

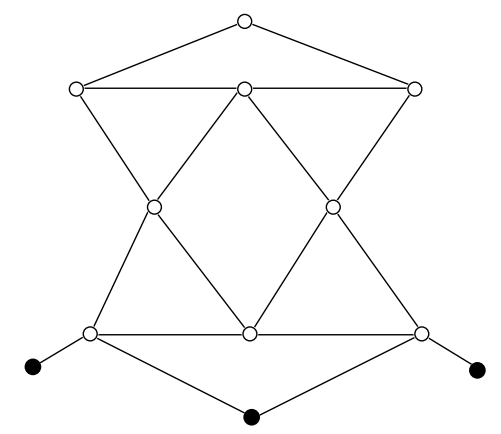

FIGURE 8. $G(36: 4,2: 3)$ on $P^{2}$.

\section{REFERENCES}

[Alperin 1980] R. Alperin, "Homology of $\mathrm{SL}_{2}(\mathbb{Z}[\omega])$ ", Comment. Math. Helv. 55:3 (1980), 364-377.

[Alperin 1987] R. C. Alperin, "An elementary account of Selberg's lemma", Enseign. Math. (2) 33:3-4 (1987), 269-273.

[Alperin 1996] R. C. Alperin, "Normal subgroups of $\mathrm{PSL}_{2}(\mathbb{Z}[\sqrt{-3}])$ ", Proc. Amer. Math. Soc. 124:10 (1996), 2935-2941.

[Bridson 1995] M. R. Bridson, "On the existence of flat planes in spaces of nonpositive curvature", Proc. Amer. Math. Soc. 123:1 (1995), 223-235.
[Brown 1997] P. Brown, Planes and non-positively curved polygons of finite groups, Ph.D. thesis, Univ. California, Berkeley, 1997.

[Brunner et al. 1985] A. M. Brunner, Y. W. Lee, and N. J. Wielenberg, "Polyhedral groups and graph amalgamation products", Topology Appl. 20:3 (1985), 289-304.

[Burnside 1911] W. Burnside, Theory of groups of finite order, 2nd ed., Cambridge Univ. Press, Cambridge, 1911. Reprinted by Dover, New York, 1955.

[Coxeter and Moser 1980] H. S. M. Coxeter and W. O. J. Moser, Generators and relations for discrete groups, 4th ed., Ergebnisse der Mathematik und ihrer Grenzgebiete 14, Springer, Berlin, 1980.

[Dicks and Dunwoody 1989] W. Dicks and M. J. Dunwoody, Groups acting on graphs, Cambridge Studies in Advanced Mathematics 17, Cambridge University Press, Cambridge, 1989.

[Floyd and Parry 1997] W. Floyd and W. Parry, "The growth of nonpositively curved triangles of groups", Invent. Math. 129:2 (1997), 289-359.

[Hsu and Wise 1998] T. Hsu and D. Wise, "Embedding theorems for nonpositively curved polygons of finite groups", Technical report, 1998. 
[Humphreys 1990] J. E. Humphreys, Reflection groups and Coxeter groups, Cambridge Studies in Advanced Mathematics 29, Cambridge University Press, Cambridge, 1990.

[Milnor 1994] J. Milnor, "How to compute volume in hyperbolic space", pp. 189-212 in Collected Papers, I: Geometry, Publish or Perish, 1994.

[Noskov 1995] G. Noskov, "Bicombings of triangle buildings", preprint, Omsk University, 1995.

[Sela 1993] Z. Sela, preprint, Hebrew University, 1993.

[Serre 1970] J.-P. Serre, "Le problème des groupes de congruence pour $\mathrm{SL}_{2}$ ", Annals of Math. 92 (1970), $489-527$.
[Serre 1971] J.-P. Serre, "Cohomologie des groupes discrets", pp. 77-169 in Prospects in mathematics (Princeton, 1970), edited by F. Hirzebruch et al., Ann. of Math. Studies 70, Princeton Univ. Press, Princeton, 1971.

[Sinkov 1936] A. Sinkov, "The groups determined by $S^{l}=T^{m}=\left(S^{-1} T^{-1} S T\right)^{p}=1$, II", Duke Math. J. 2 (1936), 74-83.

[Stallings 1991] J. R. Stallings, "Non-positively curved triangles of groups", pp. 491-503 in Group theory from a geometrical viewpoint (Trieste), edited by E. Ghys et al., World Sci. Publishing, River Edge, NJ, 1991.

Roger C. Alperin, Department of Mathematics and Computer Science, San Jose State University, San Jose, CA 95192, United States (alperin@mathcs.sjsu.edu)

Received May 2, 1997; accepted in revised form December 4, 1997 